\title{
Baubetriebliche und baurechtliche Grundlagen
}

In diesem Kapitel wird zunächst die Bedeutung der Bauzeit für die einzelnen am Bau beteiligten Parteien dargelegt, bevor die verschiedenen Bauablaufstörungen klassifiziert werden. Zudem erfolgt eine Erläuterung zu den möglichen Anspruchsgrundlagen des Auftragnehmers für eine Erstattung von Mehrkosten aus einem gestörten Bauablauf. Dieser kann in dem Verantwortungsbereich des Auftraggebers liegen oder aus höherer Gewalt resultieren.

In einem weiteren Schritt wird auf die aktuelle Rechtsprechung des Bundesgerichtshofes eingegangen und die Mindestanforderungen dargelegt, die dieser an die Nachweisbzw. Beweisführung stellt.

\subsection{Die Bedeutung der Bauzeit}

Die drei Grundsteine jedes Bauvorhabens sind: Kosten, Qualität und Zeit. Diese befinden sich naturgemäß häufig im Konflikt miteinander und werden daher oftmals als das magische Dreieck des Projektmanagements beschrieben. Da jedes Bauvorhaben einem Projekt gleicht, gilt dies auch unbeschränkt für die Baustelle.

Abhängig von dem jeweiligen Bauvorhaben sind für den Auftraggeber die einzelnen Punkte oftmals von unterschiedlicher Relevanz. Für private Bauherren stehen häufig die Kosten im Vordergrund. Größere Unternehmen, wie z. B. Energiekonzerne setzen hingegen vermehrt auf hundertprozentige Qualität, wohingegen es gerade bei Bauvorhaben der öffentlichen Hand die Bauzeit ist, die neben den Kosten am relevantesten ist. Einige Objekte, wie etwa Stadien für bestimmte Großveranstaltungen, müssen sogar zwangsläufig zu einem festgesetzten Termin fertiggestellt werden. Neben zeitlichen Zwängen durch terminliche Fixpunkte können auch steuerliche Gründe oder Finanzierungspläne auftraggeberseitig bestimmte Endtermine erfordern. 
Die Bauzeit ist allerdings nicht nur für den Auftraggeber von großer Bedeutung. Auch der Auftragnehmer trifft spätestens in der Kalkulation konkrete Annahmen über die zeitlichen Phasen einer Baustelle, um die zeitabhängigen Kosten zu erfassen. So muss ein Unternehmer sein Personal, Material und Gerät disponieren, Nachunternehmerverträge abschließen und nicht zuletzt rechtzeitig für Anschlussaufträge sorgen, um wirtschaftlich arbeiten zu können.

Die Rahmenbedingungen für die Bauzeit werden dabei oftmals bereits in der Ausschreibungsphase durch den Auftraggeber festgelegt. So werden in der Ausschreibung erfahrungsgemäß häufig zumindest Start- und Endtermin verankert. Einige Auftraggeber gehen noch einen Schritt weiter und geben bestimmte Bauphasen und Zwischentermine als sogenannte Meilensteine vor. Im Zuge der Angebotsbearbeitung ist es dann Sache des Bieters, diese Termine aufzunehmen, in kleinere Abschnitte zu gliedern und sein Angebot darauf auszulegen. Sobald folglich ein Bauvertrag geschlossen wird, wird i. d. R. auch ein Terminplan, in Form eines Bauzeitenplanes, vertraglich bindend vereinbart. Dieses zeitliche Konzept steht danach als Grundstein für alle weiteren Planungen zur Verfügung und sollte daher auch Angaben zur technischen Bearbeitung, Liefer-/ Bestell- sowie Vorlauffristen und weitere Angaben etwa zur Planfertigstellung enthalten.

Durch die vertragliche Bindung an den entwickelten Bauzeitenplan schuldet der Auftragnehmer dem Auftraggeber ,nicht nur die mangelfreie Herstellung des Bauwerks oder seiner Bauleistung, sondern gleichrangig daneben auch die fristgerechte Fertigstellung des Bauwerks" 1. Sowohl die mangelfreie Herstellung als auch die fristgerechte Fertigstellung sind rechtlich als gleichrangig anzusehen, wie anhand der $\S \S 4$ Abs. 7 und 5 Abs. 4 VOB/B deutlich wird. Hiernach kann der Auftraggeber sowohl bei mangelhafter Herstellung, als auch bei Verzug Schadensersatz geltend machen.

Da die gesamte Baustelle während der Ausführung Personal, Geräte, Nachunternehmer und weitere Hilfsmittel bindet, verursacht sie tägliche Kosten, die durch die erbrachte Leistung und die hierfür vereinbarten Preise erwirtschaftet werden müssen. Hinzu kommen allgemeine Geschäftskosten, die baustellenunabhängig als fixe Kosten in jeder Firma anfallen. Die Erwirtschaftung dieser Kosten ist nicht selten bereits bei planmäßigem Verlauf der Abläufe eine große Herausforderung für Unternehmen. Kommt es nun zu einer Störung im geplanten Ablauf, sei es durch eine Verzögerung, einen Baustopp/Stillstand, einer Beschleunigung oder durch einen anderen Grund, kann dies nicht ohne Weiteres abgefangen werden und führt unweigerlich zu Mehrkosten.

Eine weitere große Bedeutung hat die Bauzeit im Hinblick auf den Einkauf von Materialien und Nachunternehmerleistungen. Verschiedene Jahreszeiten bedingen unterschiedliche Materialpreise und Auslastungen. Wie sich aus Abb. 2.1 ergibt, variieren etwa die Preise für Betonstahl monatlich, sodass sich bei einer Verschiebung der ursprünglich geplanten Bauzeit erhebliche Mehr- und Minderkosten ergeben können.

\footnotetext{
${ }^{1}$ Vygen et al. (2002), S. 4, Rdn. 3.
} 


\section{Erzeugerpreisindex Betonstahl 2019}

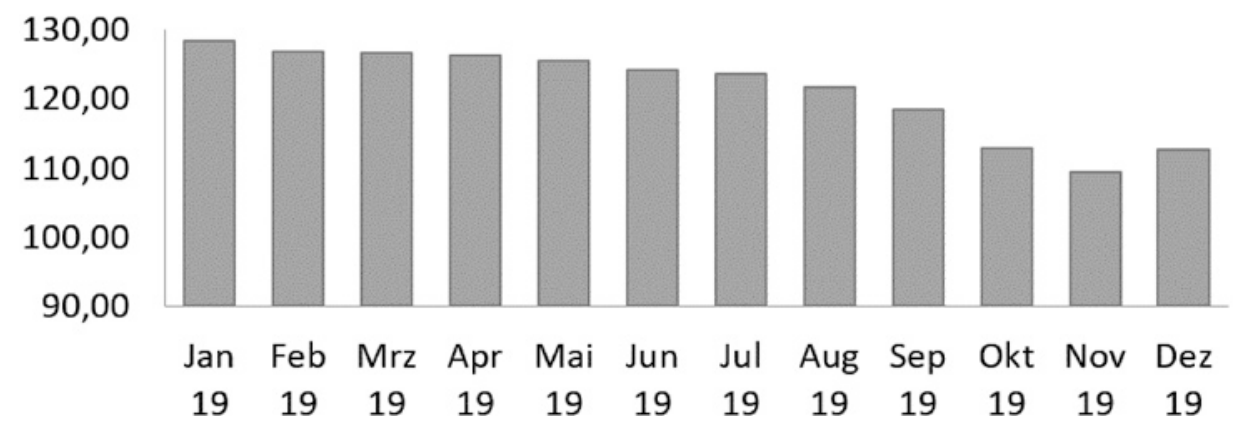

Abb. 2.1 Erzeugerpreisindex Betonstahl. (Nach den Angaben des Statistischen Bundesamtes 2020) (Die Abb. 2.1 richtet sich nach dem Index der Erzeugerpreise gewerblicher Produkte (Inlandsabsatz - Betonstahl) und bildet diesen ab. Es handelt sich nicht um den Preis pro Tonne.)

Ähnliche Schwankungen ergeben sich bei Nachunternehmerleistungen. Ist eine geplante Leistung z. B. für den Winter vereinbart, verschiebt sich nun aber aufgrund einer Bauablaufstörung in die Sommermonate, kann die Leistung aufgrund der regelmäßig höheren Auslastung im Sommer teurer werden, sodass bei dem Auftragnehmer Mehrkosten entstehen würden. Umgekehrt können Leistungen auch teurer werden, wenn eine Verschiebung in die Wintermonate erfolgt. Mehrkosten im Winter ergeben sich beispielsweise durch aus witterungsbedingten Ausfällen bzw. einer jahreszeitlich bedingten geringeren Produktivität.

Eine genaue Aufgliederung der Mehrkosten, die entweder durch eine Verlängerung resp. Verkürzung ${ }^{2}$ oder durch eine Verschiebung der Bauzeit in späteren Zeitraum entstehen können, folgt im Zuge des systematischen Kostennachweises durch den gestörten Bauablauf in Abschn. 3.4.

\subsection{Klassifizierung von Bauablaufstörungen}

Der wirtschaftliche Erfolg einer Baumaßnahme steht und fällt mit einer reibungslosen Abfolge der geplanten Schritte vom ersten Spatenstich bis zur Abnahme des Objektes. Jede eintretende Störung kann das Baustellenergebnis in Gefahr bringen, da

${ }^{2}$ Etwa durch eingeleitete Beschleunigungsmaßnahmen. 
störungsbedingte Mehrkosten schnell sehr hohe Summen erreichen können. Welche Dimensionen das Thema der Bauablaufstörung erreicht, hat Heilfort untersucht. Aus einer Unternehmensbefragung ging hervor, dass in der Baupraxis rund $56 \%$ der Vorhaben gestört waren. ${ }^{3,4}$ Dabei entstehen Mehrkosten von 30-50\% der geplanten Kosten. $^{5}$

Die Begriffe „Störung“, „Behinderung“ und „Unterbrechung“ werden in der Literatur in unterschiedlichen Zusammenhängen genutzt und sind daher an dieser Stelle genauer zu definieren, damit das nachfolgend verwendete Verständnis hinreichend deutlich wird.

Kapellmann und Schiffers definieren Störungen ,,als unplanmäßige Einwirkungen auf den [...] vertragsgemäß geplanten Produktionsprozess“" ${ }^{6}$ Dabei wird zunächst nicht unterschieden, wer ursächlich für die Störung verantwortlich ist. Die Verantwortlichkeit kann demnach auch auf der Seite des Auftragnehmers vorliegen oder durch höhere Gewalt keiner Partei zugeordnet werden können.

Born versteht in seinen Ausführungen unter Bauablaufstörungen ,unabhängig von der Verantwortlichkeit alle Störungen [...], die ein Abweichen vom ursprünglich vorgesehenen zeitlichen Ablauf der Baudurchführung bewirken, sei es, daß [sic] der vereinbarte Baubeginn verschoben, die Bauausführung ganz oder zum Teil behindert oder die Bauwerkserstellung unterbrochen wird."7

Die Ursachen von Störungen lassen sich grob in drei Verantwortungsbereiche untergliedern, die in Tab. 2.1 aufgezeigt werden.

Sobald eine Störung negative Folgen, z. B. eine terminliche Verschiebung nach sich zieht oder Mehrkosten auftreten, sprechen Kapellmann und Schiffers ${ }^{8}$ von einer Behinderung. Heiermann et al. sehen als Behinderung alle Umstände, ,die sich auf den vorgesehenen Leistungsablauf hemmend oder verzögernd auswirken. “9

In $\S 6$ Abs. 1 VOB/B heißt es:

- „Glaubt sich der Auftragnehmer in der ordnungsgemäßen Ausführung der Leistung behindert, so hat er es dem Auftraggeber unverzüglich schriftlich anzuzeigen."

Eine Behinderung im Sinne der VOB/B liegt damit dann vor, wenn die ordnungsgemäße Ausführung der Arbeiten durch den Auftragnehmer nicht oder nur beschränkt möglich

\footnotetext{
${ }^{3}$ Neuere Untersuchungen liegen dem Autor nicht vor, es ist aber davon auszugehen, dass sich der prozentuale Anteil der gestörten Bauabläufe aufgrund des gewachsenen Wissens zur Kostenaufstellung, gerade auf der Auftragnehmerseite, in den letzten Jahren weiter erhöht hat.

${ }^{4}$ Vgl. Heilfort (2001), S. 28.

${ }^{5}$ Vgl. Roquette et al. (2013), S. VII (Vorwort).

${ }^{6}$ Kapellmanns und Schiffers (2006), S. 546, Rdn. 1202.

${ }^{7}$ Born (1980), S. 45.

${ }^{8}$ Vgl. Kapellmanns und Schiffers (2006), S. 546, Rdn. 1202.

${ }^{9}$ Heiermann et al. (2011), S. 1001 (§ 6 VOB/B), Rdn. 2.
} 
Tab. 2.1 Störungsursachen. (Ergänzt nach Dreier (2001), S. 6 ff, [ohne Anspruch auf Vollständigkeit])

\begin{tabular}{l|l|l}
\hline $\begin{array}{l}\text { Ursachen aus dem Verantwortungs- } \\
\text { bereich des Auftragnehmers } \\
\text { (einschl. seiner Nachunternehmer, } \\
\text { Lieferanten, etc.) }\end{array}$ & $\begin{array}{l}\text { Ursachen aus dem Ver- } \\
\text { antwortungsbereich des } \\
\text { Auftraggebers und seiner } \\
\text { Erfüllungsgehilfen }\end{array}$ & $\begin{array}{l}\text { Ursachen außerhalb } \\
\text { der genannten Ver- } \\
\text { antwortungsbereiche }\end{array}$ \\
\hline Fehlerhafte Arbeitsvorbereitung & $\begin{array}{l}\text { Fehlende oder fehlerhafte } \\
\text { Ausführungsunterlagen }\end{array}$ & $\begin{array}{l}\text { Außergewöhnliche } \\
\text { Witterungseinflüsse }\end{array}$ \\
\hline $\begin{array}{l}\text { Fehlerhafte Beurteilung des Bauvor- } \\
\text { habens bzw. des Bauvertrages und } \\
\text { dessen Risiken }\end{array}$ & $\begin{array}{l}\text { Fehlende oder fehlerhafte } \\
\text { Genehmigungen }\end{array}$ & Streik \\
\hline $\begin{array}{l}\text { Fehlerhafte Baustelleneinrichtung } \\
\text { Zu wenig oder falsches Gerät bzw. } \\
\text { Geräteausfall }\end{array}$ & $\begin{array}{l}\text { Unwirksame Vertragsklauseln } \\
\text { stellungen }\end{array}$ & $\begin{array}{l}\text { Sämtliche Einwirkungen } \\
\text { aus ,höherer Gewalt“ }\end{array}$ \\
\hline $\begin{array}{l}\text { Mangelhafte Bau-/Projektleitung bzw. } \\
\text { schlechte Baustellen- und Nachunter- } \\
\text { nehmerbetreuung }\end{array}$ & $\begin{array}{l}\text { Nachträgliche Erhöhung } \\
\text { der Ausführungsqualitäten } \\
\text { oder erhebliche Mengen- } \\
\text { änderungen }\end{array}$ & \\
\hline $\begin{array}{l}\text { Unqualifiziertes oder zu wenig verfüg- } \\
\text { bares Personal }\end{array}$ & Mangelhafte Baü̈berwachung & \\
\hline Lieferschwierigkeiten (bei Baustoffen) & $\begin{array}{l}\text { Eingriffe in laufende Bauver- } \\
\text { fahren }\end{array}$ & \\
\hline $\begin{array}{l}\text { Zu späte Beauftragung von Nachunter- } \\
\text { nehmerleistungen }\end{array}$ & $\begin{array}{l}\text { Verspätete Fertigstellung von } \\
\text { Vorunternehmerleistungen }\end{array}$ & Störungen durch Dritte \\
\hline Mangelhafte Arbeitsausführung & Änderung des Bau-Solls & \\
\hline
\end{tabular}

ist. Allerdings gelten nach $\S 6$ Abs. 2 Nr. 2 VOB/B Witterungseinflüsse während der Ausführungszeit, mit denen bei Abgabe des Angebots normalerweise gerechnet werden müsse, nicht als Behinderung. Gleiches gilt für Krankheitsfälle, die im Rahmen der jährlichen Erkältungs- bzw. Grippewelle auftreten.

Umstände mit denen der Auftragnehmer bei Angebotsangabe zu rechnen hatte, stellen damit keine Behinderung im Sinne der VOB/B dar.

Dreier ${ }^{10}$ geht sogar noch einen Schritt weiter und trennt den Begriff in einen juristischen und einen baubetrieblichen Aspekt. Vor dem rechtlichen Hintergrund ist eine Behinderung demnach eine nähere Eingrenzung der Störung und streng mit dem Schadensersatz nach $\S 6$ Abs. 6 VOB/B verbunden. Baubetrieblich stehen eher die Abläufe auf der Baustelle im Vordergrund. Sobald in diese eingegriffen wird, z. B. durch

${ }^{10}$ Vgl. Dreier (2001), S. 9 ff. 
zusätzliche oder geänderte Leistungen gemäß $\S 2$ Abs. 5 oder Abs. 6 VOB/B, ist dies eine Störung, die zu einer Behinderung führen kann.

Diese unterschiedliche Sichtweise des Begriffes ist allerdings zu diskutieren, da beispielsweise geänderte Leistungen nicht zwangsläufig zu einer längeren Bauzeit oder Mehrkosten führen müssen, was, nach Meinung des Verfassers, im Sinne der Definition von Kapellmann/Schiffers, eine Voraussetzung für eine Behinderung sein müsste.

Wird im weiteren Verlauf von einer Störung (oder Bauablaufstörung) gesprochen, ist damit eine Störung mit finanziellen und zeitlichen Auswirkungen auf den ursprünglich geplanten Bauablauf gemeint. Der Begriff der Behinderung wird wie in $\S 6 \mathrm{Nr} .6$ VOB/B verwendet, meint also, dass die ordnungsgemäße Ausführung der Arbeiten durch den Auftragnehmer nicht oder nur beschränkt möglich ist (unter Ausschluss der einzukalkulierenden Umstände). Dies gilt ebenso für Ansprüche aus § 642 BGB.

Eine Unterbrechung liegt nach Ingenstau/Korbion dann vor, wenn der Bauablauf so weit gestört bzw. behindert wird, dass die geplanten Ausführungen zeitweise nicht mehr möglich sind, da die Unterbrechung, ,bereits nach dem allgemeinen Sprachgebrauch über den der Behinderung hinaus [geht, und einen] Arbeitsstillstand bei der Leistungsdurchführung “11 voraussetzt. Ein Sonderfall der Unterbrechung ist die Verschiebung des Baubeginns, die z. B. bei einer Bindefristverlängerung ${ }^{12}$ oder bei nicht vorliegender Ausführungsplanung (solange diese dem Auftraggeber obliegt) eintreten kann.

\subsection{Sonderfall „Höhere Gewalt"}

Einen Sonderfall der Bauablaufstörung beschreibt die höhere Gewalt.

Mit seinem Urteil VII ZR 172/86 vom 12.03.1987 hat der BGH zu einem Fall des Reiserechts geurteilt, was unter höherer Gewalt zu verstehen ist. Hier heißt es, „dass höhere Gewalt ein von außen kommendes, keinen betrieblichen Zusammenhang aufweisendes, auch durch die äußerste vernünftigerweise $\mathrm{zu}$ erwartende Sorgfalt nicht abwendbares Ereignis"13 ist. Darüber hinaus darf das eintretende Ereignis z. B. in Bezug auf seine Eintrittswahrscheinlichkeit bzw. -häufigkeit, nicht einzukalkulieren sein. Im Unterschied zu anderen Bauablaufstörungen hat im Falle höherer Gewalt keine Vertragspartei die Umstände verschuldet.

Der Fall der höheren Gewalt ist in $\S 6$ Abs. 2 Nr. 1c VOB/B geregelt. Hier heißt es: ${ }^{14}$

- „Ausführungsfristen werden verlängert, soweit die Behinderung verursacht ist [...] durch höhere Gewalt oder andere für den Auftragnehmer unabwendbare Umstände. “

\footnotetext{
${ }^{11}$ Ingenstau und Korbion (2010), S. 1341, Rdn. 3.

${ }^{12}$ Vgl. Mitschein A. (1999), S. $69 \mathrm{ff}$.

${ }^{13}$ BGH-Urteil VII ZR 172/86 vom 12.03.1987.

${ }^{14}$ VOB (2019), Teil B $§ 6$ Abs. 2.
} 
Und weiter in $\S 6$ Abs. 2 Nr. 2 VOB/B: $:^{15}$

- „Witterungseinflüsse während der Ausführungszeit, mit denen bei Abgabe des Angebots normalerweise gerechnet werden musste, gelten nicht als Behinderung. “

Kommt es folglich bei Baustellen zu Behinderungen des Auftragnehmers aus Gründen, deren Ursache der höheren Gewalt zuzuordnen ist, hat der Auftragnehmer einen Anspruch auf entsprechende Bauzeitverlängerung, deren Berechnung in Abschn. 3.3 folgt.

Im Frühjahr 2020 hat sich das Virus Sars-CoV-2 bzw. die dazugehörige Krankheit Covid-19, besser bekannt unter dem Begriff „Corona“ weltweit ausgebreitet und wurde im März 2020 von der Weltgesundheitsorganisation WHO als Pandemie, also als weltweit geltende und auftretende Seuche, eingestuft. Vor diesem Hintergrund sind zunehmend auch Bauunternehmen und deren Baustellen von Covid-19 betroffen.

Unter Betrachtung des oben genannten $\S 6$ Abs. 2 Nr. 2 VOB/B ist in Krankheitsfällen $\mathrm{zu}$ beachten, dass beispielsweise Erkrankungen von einzelnen Beschäftigten, wie etwa bei einer ,normalen“ Grippewelle, einzukalkulieren sind und damit nicht den Anforderungen an $\S 6$ Abs. 2 Nr. 1c VOB/B genügen. Vielmehr müsste ein Ausfall von Beschäftigten Ausmaße annehmen, die über ein kalkulierbares Maß hinaus gehen, um als höhere Gewalt eingestuft zu werden.

Ein solch unkalkulierbares Ereignis tritt bspw. ein, wenn weitgehende Quarantänemaßnahmen für die Mehrzahl der Beschäftigten ausgesprochen werden, Material- oder Lieferketten überregional ausfallen oder es zu unabsehbaren Beeinträchtigungen im Verhältnis zu eigenen Nachunternehmern kommt. Einzelne Fälle lokaler Quarantänemaßnahmen oder ein Nichteinsatz eines Arbeitnehmers als Vorsichtsmaßnahme reichen allerdings nicht aus, um als höhere Gewalt eingestuft zu werden. Anders verhält es sich, wenn mehrere Beschäftigte behördlicherseits unter Quarantäne gestellt oder gar ein Arbeitsverbot auferlegt wird.

Aktuell gibt es noch keine baurechtliche Rechtsprechung zu dieser Thematik. Mit Verweis auf das Reiserecht, ist davon auszugehen, dass Einschränkungen durch Covid-19 nicht allein in den Risikobereich des Bauunternehmens fallen. Betroffene Bauunternehmen sollten für jeden Einzelfall individuell dokumentieren wann es, durch welchem Umstand, zu welcher Behinderung gekommen ist und mit welchen Folgen zu rechnen ist. Vorsorglich und allgemein gefasste Behinderungsanzeigen dienen dem Bauherrn nur als Information und sind rechtlich nicht bedeutsam.

Anders verhält es sich wiederum, wenn der Auftraggeber aus Gründen einer Pandemie seiner Mitwirkungspflicht nicht nachkommen kann. In diesem Fall fällt die Behinderung in den Risikobereich des Bauherrn. Dies ist beispielsweise der Fall, wenn

\footnotetext{
${ }^{15}$ VOB (2019), Teil B § 6 Abs. 2.
} 
der Auftraggeber (oder dessen Vertreter) für den Bauablauf unabdingbare Termine nicht einhalten kann oder etwa Vorleistungen nicht rechtzeitig erbracht wurden.

Bei Bauverträgen auf Grundlage des BGB kommt bei Fällen höherer Gewalt eine Störung der Geschäftsgrundlage in Betracht ( $\$ 313$ BGB) sowie gegebenenfalls auch Entschädigung während des Annahmeverzugs des Bauherrn (§ 642 BGB).

In jedem Fall ist eine sorgfältige Dokumentation der einzelnen Umstände wichtig, wenn es $\mathrm{zu}$ einer Behinderung durch höhere Gewalt kommt. Hierbei wird ein allgemeiner Hinweis auf Covid-19 nicht ausreichen. Vielmehr bedarf es Nachweisen in jedem Einzelfall und für jede Baustelle.

Da im Falle der höheren Gewalt kein Vertragspartner für die Störung verantwortlich ist, bedarf es in solcher Situation einer maximalen Transparenz und Kommunikation aller Vertragspartner über konkrete, baustellenbezogene Störungssachverhalte. Dies kann unter anderem bedeuten, dass gemeinsam eine baustellenbezogene Risikoabschätzung und Fortführungsbewertung geführt werden sollte.

Sobald es durch höhere Gewalt zu Bauablaufstörungen kommt, sind diese dem Vertragspartner schriftlich anzuzeigen. Dafür ist eine direkte, baustellenbezogene Kommunikation, in der Regel in Form eines Behinderungsschreibens nach $\S 6$ Abs. 1 $\mathrm{VOB} / \mathrm{B}$, erforderlich.

Dabei bleibt $\mathrm{zu}$ beachten, dass derjenige Vertragspartner, der sich auf eine Behinderung durch die Umstände höherer Gewalt beruft, voll beweisbelastet ist. Auftragnehmer müssen demnach die Umstände der höheren Gewalt darlegen und beweisen bzw. nachvollziehbar begründen, warum und in welchem Umfang sie sich in der vorherrschenden Situation außerstande sehen, ihren Leistungspflichten weiterhin nachzukommen.

\subsection{Anspruchsgrundlagen für Mehrkostenansprüche}

Bauablaufstörungen können, wie in Tab. 2.1 (Abschn. 2.2) dargestellt ist, aus verschiedenen Einflussbereichen heraus entstehen. Die auftraggeberseitigen Störungsursachen sind in der Praxis auf drei Hauptursachen zurückzuführen. Zum einen kann der Auftraggeber im Sinne der vertraglichen Regelungen nach $\S 1$ Abs. 3 und $\S 1$ Abs. 4 VOB/B Anordnungen treffen, wodurch zusätzliche bzw. geänderte Leistungen entstehen. In einem solchen Fall ist eine Vereinbarung über die Vergütung der Leistung zu treffen bzw. der Auftragnehmer hat einen Anspruch auf besondere Vergütung nach $\S 2$ Abs. 5 bzw. Abs. 6 VOB/B. Zum Zweiten kann es zu einer Behinderung gemäß $\$ 6 \mathrm{VOB} / \mathrm{B}$ kommen. In diesem Fall kann von dem Vertragspartner, der die Behinderung nicht zu vertreten hat, Schadensersatz nach $\S 6$ Abs. 6 VOB/B verlangt werden. Drittens kann es sich um eine Verletzung der Mitwirkungspflichten des Auftraggebers handeln. Dazu gehört auch der Fall, dass Vorunternehmerleistungen nicht rechtzeitig abgeschlossen 
werden. Der Auftraggeber gerät dann in Annahmeverzug ${ }^{16}$ nach $\S \S 293$ ff. BGB. Der Auftragnehmer kann dann eine angemessene Entschädigung nach §642 BGB verlangen ${ }^{17}$.

\subsubsection{Vergütung gemäß § 2 Abs. 3, Abs. 5 und Abs. 6 VOB/B}

Die VOB stellt ein Vertragswerk dar, welches die gegenläufigen Interessenlagen der Bauherrenseite und der Unternehmen berücksichtigen und in Ausgleich bringen soll.

Nach $\S 2$ Abs. 3, 5 und 6 VOB/B hat der Auftragnehmer einen Anspruch auf zusätzliche Vergütung für Mehr-/Mindermengen und geänderte bzw. zusätzliche Leistungen. Diese Rechte des Auftragnehmers stellen das Gegenstück zu dem Leistungsanordnungsrecht des Auftraggebers aus $\S 1$ Abs. 3 und Abs. 4 VOB/B dar, die es dem Auftraggeber erlauben, Anordnungen zu Änderungen des Bauentwurfes oder zu nicht vereinbarten, aber für die Bauausführung notwendigen Leistungen zu treffen. ${ }^{18}$

Mehr- oder Mindermengen gemäß $\S 2$ Abs. 3 VOB/B ergeben sich während der Bauausführung von selber. Sie sind jedoch hier zu erwähnen, da sehr große Mehrmengen erhebliche Auswirkungen auf die Bauzeit haben können. Zudem kann in bestimmten Fallkonstellationen ein Anspruch auf Vereinbarung eines neuen Preises gegeben sein.

Sofern der Auftraggeber durch Änderungen des Bauentwurfs eine Änderung der im Vertrag vorgesehenen Leistung verlangt, muss er dies ausdrücklich anordnen.

Im $\S 2$ der VOB/B heißt es in den Absätzen 5 und 6:19

- „(5) Werden durch Änderung des Bauentwurfs oder andere Anordnungen des Auftraggebers die Grundlagen des Preises für eine im Vertrag vorgesehene Leistung geändert, so ist ein neuer Preis unter Berücksichtigung der Mehr-oder Minderkosten zu vereinbaren. Die Vereinbarung soll vor der Ausführung getroffen werden. “

- „(6) 1. Wird eine im Vertrag nicht vorgesehene Leistung gefordert, so hat der Auftragnehmer Anspruch auf besondere Vergütung. Er muss jedoch den Anspruch dem Auftraggeber ankündigen, bevor er mit der Ausführung der Leistung beginnt.

- (6) 2. Die Vergütung bestimmt sich nach den Grundlagen der Preisermittlung für die vertragliche Leistung und den besonderen Kosten der geforderten Leistung. Sie ist möglichst vor Beginn der Ausführung zu vereinbaren. “

\footnotetext{
${ }^{16}$ In Annahmeverzug gerät der Auftraggeber dann, wenn er die angebotene Leistung des Auftragnehmers nicht annimmt, obwohl der Auftragnehmer die Leistung ordnungsgemäß anbietet. §§ $293 \mathrm{ff}$. BGB.

${ }^{17}$ Vgl. Bieber (2009), S. 12, Folien 23-24.

${ }^{18}$ Vgl. VOB (2019), Teil B § 1 Abs. 3 und Abs. 4.

${ }^{19}$ Vgl. VOB (2019), Teil B $§ 2$ Abs. 5 und Abs. 6.
} 
Unter der „Änderung des Bauentwurfes“ sind „sämtliche Anordnungen, die die Art und den Umfang der vertraglich festgelegten Leistung betreffen“20 zu verstehen. Der Begriff ist dabei nicht auf Planungsänderungen begrenzt.

Eine vom Vertrag abweichende Vergütung steht dem Auftragnehmer demnach immer dann zu, sobald sich die „Grundlagen des Preises für eine im Vertrag vorgesehene Leistung“21 ändern oder eine zusätzliche Leistung durch den Auftraggeber gefordert wird. ${ }^{22}$

Bei Ansprüchen auf Vergütung durch eine zusätzliche Leistung nach $\S 2$ Abs. 6 VOB/B sieht die VOB/B vor, dass der Auftragnehmer seinen Anspruch auf die Erstattung der aufgewendeten Mehrkosten vor Beginn der Arbeiten ankündigt. ${ }^{23}$

Eine entsprechende Regelung ist bei der Anpassung der Vergütung nach § 2 Abs. 5 VOB/B nicht vorgesehen. Allerdings ist geregelt, dass ein neuer Preis unter Berücksichtigung der Mehr- oder Minderkosten zu vereinbaren ist, möglichst bevor mit der Ausführung begonnen wird. ${ }^{24}$ Durch diese vorher zu treffende Vereinbarung wird sichergestellt, dass Auftraggeber und -nehmer sich über die Mehrkosten verständigen. Eine Vereinbarung zwischen den Vertragsparteien setzt allerdings ebenfalls eine Verständigung über Mehrkosten voraus, die erfordert, dass der Auftragnehmer den Auftraggeber über etwaige entstehende Mehrkosten in Kenntnis setzt. Ähnlich sieht es auch Vygen. Er fordert, dass eine Ankündigung der Mehrkosten im Falle der Vergütungsanpassung nach $\S 2$ Abs. $5 \mathrm{VOB} / \mathrm{B}$ erfolgen soll, wodurch nicht mehr unterschieden werden müsse, auf welchen der beiden Paragraphen sich die Vertragspartner berufen. ${ }^{25}$

Eine Differenzierung zwischen der geänderten und der zusätzlichen Leistung fällt aus baubetrieblicher Sicht insofern leichter, als dass dem Auftraggeber in beiden Fällen die Mehrkosten angemeldet werden und die Berechnung der Mehrkosten auf Grundlage des Preises bzw. der Preisermittlung ${ }^{26}$, sprich der Urkalkulation, erfolgt. Da die Berechnung der Anspruchshöhe in beiden Fällen ähnlich ist, werden hier beide Punkte gemeinsam dargestellt. Vergütet werden nur die Mehr- oder Minderkosten auf Basis der Urkalkulation als Fortschreibung der Preisermittlungsgrundlage für die vertraglich festgelegten Positionen. Die tatsächlich anfallenden Kosten sind nicht maßgebend. ${ }^{27}$

Anordnungen vonseiten des Auftraggebers setzen eine aktive Handlung seinerseits voraus. Geht es um einfache geänderte oder zusätzliche Leitungen, die klar

\footnotetext{
${ }^{20}$ Fischer et al. (2001), S. 46.

${ }^{21}$ Vgl. VOB (2019), Teil B § 2 Abs. 5.

${ }^{22}$ Vgl. VOB (2019), Teil B $\S 2$ Abs. 6.

${ }^{23}$ Vgl. VOB (2019), Teil B $§ 2$ Abs. 6.

${ }^{24}$ Vgl. VOB (2019), Teil B $§ 2$ Abs. 5.

${ }^{25}$ Vgl. Vygen et al. (2002), S. 140, Rdn. 187.

${ }^{26}$ Vgl. VOB (2019), Teil B § 2 Abs. 5 und Abs. 6.

${ }^{27}$ Vgl. Bieber (2009) S. 12 ff, Folien 23-26.
} 
definiert sind, kommt es hierbei kaum zu Streitigkeiten. Der Auftraggeber wünscht eine Änderung, ordnet diese an, woraufhin der Auftragnehmer Mehrkosten anmeldet. Sofern der Auftraggeber diese akzeptiert, führt der Auftragnehmer die Anordnung aus und erhält im Gegenzug seine Vergütung.

Mehrkostenansprüche, die durch Bauablaufstörungen entstehen, gestalten sich komplexer. Hier ist zunächst zu überprüfen, ob eine Störung der Arbeiten aus einer Anordnung durch den Auftraggeber entstammt. Vygen ist der Meinung, dass dies im Allgemeinen zwar nicht der Fall sei, sobald Störungen aber ursächlich z. B. auf Änderungen des Bauentwurfs oder der technischen Ausführung zurückzuführen sind, diese nach $\S 2$ Abs. 5 abzurechnen seien. ${ }^{28}$

Unter Änderungen des Bauentwurfs fallen auch alle Änderungen, die sich auf die vertraglich geregelten Rahmenbedingungen beziehen. Dies können sowohl Änderungen des Bauzeitenplans, als auch Änderungen der Statik oder anderer Pläne, die in dem Verantwortlichkeitsbereich des Auftraggebers liegen, sein, wie auch der BGH 2014 festgelegt hat:

- „Die Regelung des $\$ 2$ Nr. 5 VOB/B kommt auch zur Anwendung, wenn die Verlängerung der Bauzeit auf eine Maßnahme des Auftraggebers zurückzuführen ist, die ihre Ursache in seinem Risikobereich hat. Diese Voraussetzung ist erfüllt, wenn sich die Bauzeit dadurch verzögert, dass der Auftraggeber die von ihm geschuldete Statik und die Planung nicht rechtzeitig vorlegt. "29

Eine weitere wichtige Entscheidung bezüglich Bauablaufstörungen hat der BGH im Jahr $2009^{30}$ getroffen, in der es heißt, dass sich durch ein verzögertes Vergabeverfahren erstens zusätzliche Bauzeit ergäbe und zweitens, die durch die Verzögerung entstehenden Mehrkosten in Anlehnung an die Grundsätze des $§ 2$ Nr. 5 VOB/B anzupassen seien. Dieses Urteil ist im Hinblick auf die Bindefristverlängerung als Sonderfall in Abschn. 3.4.4 von großer Bedeutung und wird dort ausführlicher behandelt.

Eine genauere Erläuterung der wichtigsten Urteile in Bezug auf Bauablaufstörungen folgt in Abschn. 2.5.1.

\subsubsection{Schadensersatz gemäß § 6 Abs. 6 VOB/B}

Während sich eine Vergütung rein auf die Mehr- oder Minderkosten auf kalkulativer Basis bezieht, geht es bei der Anspruchsgrundlage des Schadensersatzes nach $§ 6$ Abs. 6

\footnotetext{
${ }^{28}$ Vgl. Vygen et al. (2002), S. 121, Rdn. 162.

${ }^{29}$ BGH-Urteil VII ZR 141/12 vom 08.05.2014

${ }^{30}$ Vgl. BGH-Urteil VII ZR 11/08 vom 11.05.2009.
} 
VOB/B um die nachweislich entstandenen Mehrkosten durch eine Behinderung ${ }^{31}$ oder Unterbrechung. $§ 6$ Abs. 6 VOB/B lautet: ${ }^{32}$

- „Sind die hindernden Umstände von einem Vertragsteil zu vertreten, so hat der andere Teil Anspruch auf Ersatz des nachweislich entstandenen Schadens, des entgangenen Gewinns aber nur bei Vorsatz oder grober Fahrlässigkeit. Im Übrigen bleibt der Anspruch des Auftragnehmers auf angemessene Entschädigung nach \$ 642 BGB unberührt, sofern die Anzeige nach Absatz 1 Satz 1 erfolgt oder wenn Offenkundigkeit nach Absatz 1 Satz, 2 gegeben ist. “

Auf Rechtsfolgenseite ist nach $§ 249$ BGB der Zustand herzustellen, ,der bestehen würde, wenn der zum Ersatz verpflichtende Umstand nicht eingetreten wäre“. ${ }^{33}$ Es können demnach nach der sogenannten Differenztheorie alle Mehrkosten geltend gemacht werden, die in der störungsbedingten Ist-Situation mehr anfallen als in der hypothetischen Soll-Situation entstanden wären, wenn es nicht zu einer Störung gekommen wäre. ${ }^{34}$

Dies umfasst im Wesentlichen Stillstandskosten, zeitabhängige Mehrkosten, und allgemeine Geschäftskosten ${ }^{35}$ für die gesamte Dauer der Behinderung. Die Erstattung des ausgebliebenen Gewinns sieht die VOB/B allerdings nur bei grober Fahrlässigkeit oder Vorsatz vor. ${ }^{36}$ Für die Ermittlung der entstandenen Kosten können Rechnungen, z. B. für Gerätemieten oder von Nachunternehmern, herangezogen werden. Schwieriger wird es bei firmeneigenen Geräten und Materialien. In diesem Fall können Richtwerte gemäß der Baugeräteliste (BGL) angesetzt werden.

Damit Schadensersatzansprüche geltend gemacht werden können, müssen bestimmte Voraussetzungen erfüllt sein. Der Auftraggeber muss als Vertragspartner aufgrund einer schuldhaften Vertragsverletzung für die Störung des Ablaufes, die zu einer Behinderung mit zeitlichen und/oder finanziellen Folgen führt, verantwortlich sein, und der Auftragnehmer muss diese Behinderung gemäß $§ 6$ Abs. 1 VOB/B anmelden, wobei eine Anmeldung bei Offenkundigkeit entbehrlich ist. ${ }^{37}$ Zudem muss der Schaden nachweisbar auf die Störung zurückzuführen sein. Den Nachweis für das Vorliegen der Voraussetzungen hat der Auftragnehmer zu erbringen.

\footnotetext{
${ }^{31}$ Wie in Abschn. 2.2 beschrieben, liegt eine Behinderung immer dann vor, wenn es durch eine Störung zu negativen zeitlichen und/oder finanziellen Folgen kommt.

${ }^{32} \mathrm{VOB}(2019)$, Teil B $\S 6$ Abs. 6.

${ }^{33}$ BGB (2020), § 249 Abs.1.

${ }^{34}$ Vgl. Kumlehn (2004), S. 28.

${ }^{35} \mathrm{Vgl}$. Leineweber (2002), S. 135.

${ }^{36}$ Vgl. VOB (2019), Teil B § 6 Abs. 6.

${ }^{37}$ Eine Anmeldung der Behinderung ist nur entbehrlich, wenn Offenkundigkeit vorliegt. Weiterführend zum Begriff der Offenkundigkeit: Kapellmann und Schiffers (2006), S. 554 f, Rdn. 1221 f.
} 
Tab. 2.2 Anforderungen an eine Behinderungsanzeige

\begin{tabular}{|c|c|}
\hline \multicolumn{2}{|c|}{ Behinderungsanzeige } \\
\hline \multirow[t]{4}{*}{ Zeitpunkt } & Unverzüglich \\
\hline & Ohne schuldhaftes Zögern \\
\hline & Sobald sich der Auftragnehmer behindert „glaubt“ \\
\hline & Ein Eintritt der Behinderung ist nicht notwendig \\
\hline Form & Schriftlich, gem. $\S 6$ Abs. 1 Nr. 1 VOB/B \\
\hline Adressat & Auftraggeber bzw. dessen rechtlicher Vertreter \\
\hline \multirow[t]{4}{*}{ Inhalt } & Tatsache und Wirkung \\
\hline & Grund (Welche maßgeblichen Umstände sind bekannt?) \\
\hline & Hindernde Wirkung (Was sind die Auswirkungen?) \\
\hline & Voraussichtliche Dauer \\
\hline
\end{tabular}

Neben seinem eigenen Verschulden hat der Auftraggeber auch für das Verschulden seiner Erfüllungsgehilfen einzustehen. ${ }^{38}$ Beispiele hierfür sind verspätete Planlieferungen oder verspätete Freigabe von Musterobjekten zu nennen, ohne die nicht weitergearbeitet werden kann. Hierzu zählen auch auftraggeberseitig bedingte Bauzeitverschiebungen ${ }^{39}$ oder fehlerhafte bzw. unzureichende Gutachten bspw. über den Baugrund.

Die oben angesprochene Behinderungsanzeige muss für die erfolgreiche Durchsetzung von Mehrkostenerstattungsansprüchen gewissen formalen und inhaltlichen Anforderungen entsprechen, die in Tab. 2.2 näher dargelegt sind.

Die Behinderungsanzeige sollte demnach unverzüglich schriftlich erfolgen und sich an den Auftraggeber als Vertragspartner richten. Inhaltlich muss dem Auftraggeber der kausale Zusammenhang zwischen Störung und deren Auswirkung in Form von Mehrkosten aufgezeigt werden, ohne sich dabei auf eine konkrete Schadenshöhe oder die Dauer der Behinderung festzulegen. Sie dient dem Auftraggeber als Information und soll ihm Schutz bieten, bzw. warnen und die Möglichkeit geben, die drohenden Behinderungen auszuräumen. ${ }^{40}$

Sollte ein Auftragnehmer im Streitfall nicht beweisen können, dass er seiner aus $§ 6$ Abs.1 VOB/B folgenden Pflicht zur schriftlichen Anzeige einer Behinderung, nachgekommen ist, wird er keinerlei Ansprüche durchsetzen können.

Zu beachten ist dabei, dass die Angabe der Höhe eines etwaigen Ersatzanspruches nicht Inhalt der Behinderungsanzeige sein muss.

\footnotetext{
${ }^{38}$ Ingenstau und Korbion (2010), S. 1380, Rdn. 13.

${ }^{39}$ Urteil 20 U 164/84 des OLG Köln vom 14.06.1985.

${ }^{40}$ Vgl. BGH-Urteil VII ZR 185/98 vom 21.10.1999.
} 


\subsubsection{Entschädigung gemäß § 642 BGB}

Eine weitere Anspruchsgrundlage für Mehrkosten infolge von Bauablaufstörungen ist die Entschädigung gemäß $§ 642$ BGB, in welchem es heißt: ${ }^{41}$

- „Ist bei der Herstellung des Werkes eine Handlung des Bestellers erforderlich, so kann der Unternehmer, wenn der Besteller durch das Unterlassen der Handlung in Verzug der Annahme kommt, eine angemessene Entschädigung verlangen. “

Dass die Regelungen des BGB auch bei einem VOB-Vertrag Anwendung finden, hat der BGH in seinem sog. „,Vorunternehmer II“-Urteil ${ }^{42}$ vom 21.10.1999 (Az. VII ZR 185/98) entschieden. Näheres hierzu folgt in Abschn. 2.5.1.

$\S 642$ Abs. 1 BGB befasst sich mit einem Entschädigungsanspruch des Unternehmers (entspricht dem Auftragnehmer) gegenüber dem Besteller (entspricht dem Auftraggeber), wenn dieser eine Mitwirkungshandlung unterlässt, wodurch der Auftraggeber in Annahmeverzug kommt. Der größte Unterschied zu $§ 6$ Abs. 6 VOB/B ist hierbei, dass $\S 642$ BGB kein Verschulden voraussetzt, wodurch sich auch „die Fälle der Behinderung des Auftragnehmers durch verspätete oder mangelhafte Vorunternehmerleistungen, auf die der Nachfolgeunternehmer bei seiner Bauausführung angewiesen ist, angemessen und gerecht lösen“ ${ }^{\star 43}$ lassen. Der Anspruch ist allerdings ,,nicht auf diese bisher nicht angemessen zu lösenden Fälle beschränkt, sondern [könne] auch dann herangezogen werden, wenn der Schadensersatzanspruch aus $\S 6$ Abs. 6 VOB/B am fehlenden Verschulden des Auftraggebers scheitert “44. Dies lässt dem Auftragnehmer die Wahl der Anspruchsgrundlage offen.

Auch wenn §642 BGB verschuldensunabhängig ist, wird eine unterlassene Mitwirkungshandlung des Auftraggebers vorausgesetzt. Diese können u. a.

- die rechtzeitige und mangelfreie Lieferung von bauseits zu liefernden Baumaterialien, Plänen, und Leistungen,

- die rechtzeitige Bereitstellung von Baugenehmigungen und anderen erforderlichen Genehmigungen,

- die rechtzeitige Entscheidung über die Ausführung von Alternativen und Bemusterungen,

- die Erfüllung seiner Pflichten nach $\S 4$ Abs. 1 VOB/B

$\operatorname{sein}^{45}$

${ }^{41}$ Vgl. BGB (2020), § 642.

${ }^{42}$ In diesem Urteil geht es um den Anspruch auf Schadensersatz wegen Behinderung durch verspätet fertiggestellte Vorunternehmer. Es revidiert die bis dahin geltende Rechtsprechung des BGH Urteils VII ZR 23/84 vom 27.06.1985 („Vorunternehmer I“).

${ }^{43}$ Vygen et al. (2002), S. 231, Rdn. 313.

${ }^{44}$ Vygen et al. (2002), S. 231, Rdn. 313.

${ }^{45}$ Vgl. Vygen et al. (2002), S. 232, Rdn. 314. 
Die Höhe des Entschädigungsanspruchs ist in $\S 642$ Abs. 2 BGB geregelt. Dieser richtet sich nach der vereinbarten Vergütung ${ }^{46}$, und wirft somit die Frage auf, wo der Unterschied zwischen Schadensersatz und Entschädigung liegt.

Wie in Abschn. 2.4.2 erläutert, berechnet sich der zu ersetzende Schaden i.S. des $\S 6$ Abs. $6 \mathrm{VOB} / \mathrm{B}$ nach der Differenztheorie zwischen der behinderungsbedingten Situation und der hypothetisch ursprünglich geplanten Situation ohne Behinderung und wird damit zum Ausgleich der tatsächlichen Mehraufwendungen. Bei der Entschädigung hingegen ist die vereinbarte Vergütung als Grundlage genannt. Sie bezieht sich demnach auf den vertraglich vereinbarten Preis und damit auch auf die Urkalkulation, die fortgeschrieben werden darf. Dies heißt im Umkehrschluss, dass die Entschädigung nicht auf tatsächliche Mehrkosten zurückzuführen ist, sondern auch Kosten beinhalten kann, die entstehen, wenn keine Aufwendungen verursacht werden, sondern es zu Stillständen kommt. ${ }^{47}$ So sieht es auch das Kammergericht Berlin welches entschieden hat, dass „über $§ 642$ BGB [...] wartezeitbedingte Mehrkosten des Unternehmers entschädigt“ 48 werden können.

Nicht eindeutig geregelt ist die Frage nach dem entgangenen Gewinn bei einem Entschädigungsanspruch. Der BGH verweist in seinem Urteil „Vorunternehmer II“ darauf, dass dieses nicht entgangenen Gewinn und Wagnis umfasse ${ }^{49}$. Dieser Punkt ist in der aktuellen Literatur ${ }^{50}$ allerdings sehr umstritten, da der entgangene Gewinn und das Wagnis zweifellos zur vereinbarten Vergütung und auch in der bisherigen höchstrichterlichen Rechtsprechung zu einem Entschädigungsanspruch gehören ${ }^{51}$.

Der Anspruch auf Entschädigung setzt einen Annahmeverzug des Auftraggebers voraus. Dies ist nach $\S 293$ BGB dann der Fall, wenn der Auftraggeber die ihm, durch den Auftragnehmer angebotene Leistung nicht annimmt. Um Entschädigungsansprüche geltend machen zu können, muss der Auftragnehmer folglich, neben der Behinderungsanzeige, seine Leistungsbereitschaft zeigen.

\footnotetext{
${ }^{46}$ Vgl. BGB (2020), § 642 Abs. 2.

${ }^{47}$ Vgl. Kapellmann und Schiffers (2006), S. 762, Rdn. 1649.

${ }^{48}$ Vgl. Urteil 7 U 12/12 des KG Berlin vom 28.05.2013.

${ }^{49}$ BGH-Urteil VII ZR 185/98 vom 21.10.1999.

${ }^{50}$ Vgl. hierzu unter anderem: Vygen et al. (2002), S. 237 ff, Rdn. 324; Kapellmann und Schiffers (2006); Rdn. 1650: „Es gibt keinen Grund, dann, wenn man kraft Gesetzes an die ,Höhe der vereinbarten Vergütung' anknüpfen muss, willkürlich aus der ,vereinbarten Vergütung ' einen Bestandteil [...] zu streichen [...] Die Streichung des Gewinns bei § 642 wäre ,Strafe ' und alles andere als , angemessene "Entschädigung“; Döring in Ingenstau und Korbion (2010); S. 1399, Rdn. 61/62.

${ }^{51}$ Vgl. Kapellmann und Schiffers (2006), S. 764, Rdn. 1650.
} 


\subsubsection{Vergleich und Wahl der Anspruchsgrundlagen}

Abhängig von der Ursache für die Störung stehen dem Auftragnehmer wie dargelegt drei Anspruchsgrundlagen zur Verfügung, vorausgesetzt alle Anspruchsvoraussetzungen sind erfüllt.

In der Baupraxis ist es bei Bauablaufstörungen aufgrund verschiedener Gemengelagen von Vorkommnissen häufig nicht einfach möglich, die Störungsursachen und die daraus folgenden Anspruchsgrundlagen zu trennen. Hinzu kommt, dass ein erheblicher Unterschied in der Nachweisführung zwischen den Mehrkostenansprüchen aus Vergütung und denen aus Schadensersatzforderungen vorhanden ist, wodurch Auftragnehmer gerne eine Vergütung bzw. Entschädigung geltend machen, während vom Auftraggeber tendenziell eher ein Schadensersatznachweis gefordert wird. ${ }^{52}$

Darüber hinaus entstehen bei der Berechnung der Schadenersatzansprüche nach $\S 6$ Abs. 6 VOB/B in der Praxis aus baubetrieblicher Sicht oft Schwierigkeiten, da sich diese nach der Differenztheorie (siehe Abschn. 2.4.2) aus der hypothetisch ohne Schaden eingetretenen und der realen Vermögenslage berechnen, eine Baustelle aber nur in den seltensten Fällen wie geplant abläuft, wodurch die hypothetisch ohne Schaden eingetretene Vermögenslage schwer zu ermitteln ist und Streitpotenzial birgt. ${ }^{53}$

Mitschein ist der Ansicht, dass es ein praktikabler Ansatz wäre, störungsbedingte Kostenermittlungen aus Schadensersatzansprüchen und aus Vergütungsansprüchen nach dem gleichen Schema zu ermitteln, wofür es eine rechtliche Grundlage geben sollte. ${ }^{54}$

Da eine solche rechtliche Grundlage bislang nicht gegeben ist, versuchen Auftragnehmer häufig ihre Schadensersatzansprüche aus $\S 6$ Abs. 6 VOB/B eher als Entschädigung gemäß $§ 642$ BGB und damit vergütungsähnlich durchzusetzen. Dass dies möglich ist, hat der BGH 1999 entschieden. Hier heißt es im Urteil VII ZR 185/98 vom 21.10.: „§ 642 BGB wird durch $\S 6$ Nr. 6 VOB/B nicht verdrängt ${ }^{\text {“55 }}$, woraufhin auch die VOB geändert wurde. Ab der Version von 2006 heißt es in $\S 6$ Abs. 6 Satz Nr. 2 VOB/B: „Im Übrigen bleibt der Anspruch des Auftragnehmers auf angemessene Entschädigung nach $\S 642$ BGB unberührt ${ }^{\star 56}$.

Tab. 2.3 zeigt einen Überblick bzw. eine Gegenüberstellung der Anspruchsgrundlagen sowie der Grundlagen der Entschädigung-, Vergütungs- und Schadensermittlung.

Auch wenn sich die Anspruchsgrundlagen in der Ermittlung der Höhe stark unterscheiden können, gibt es gemeinsame Punkte. So sind beispielsweise Parallelen in Bezug auf die Darstellung der Auswirkungen auf den Bauablauf zu sehen.

\footnotetext{
${ }^{52}$ Vgl. Dreier (2001), S. 110.

${ }^{53}$ Vgl. Dreier (2001), S. 2.

${ }^{54} \mathrm{Vgl}$. Mitschein (1999), S. 120.

${ }^{55}$ BGH-Urteil VII ZR 185/98 vom 21.10.1999.

${ }^{56}$ Vgl. VOB (2019), Teil B § 6 Abs. 6 Nr. 2.
} 


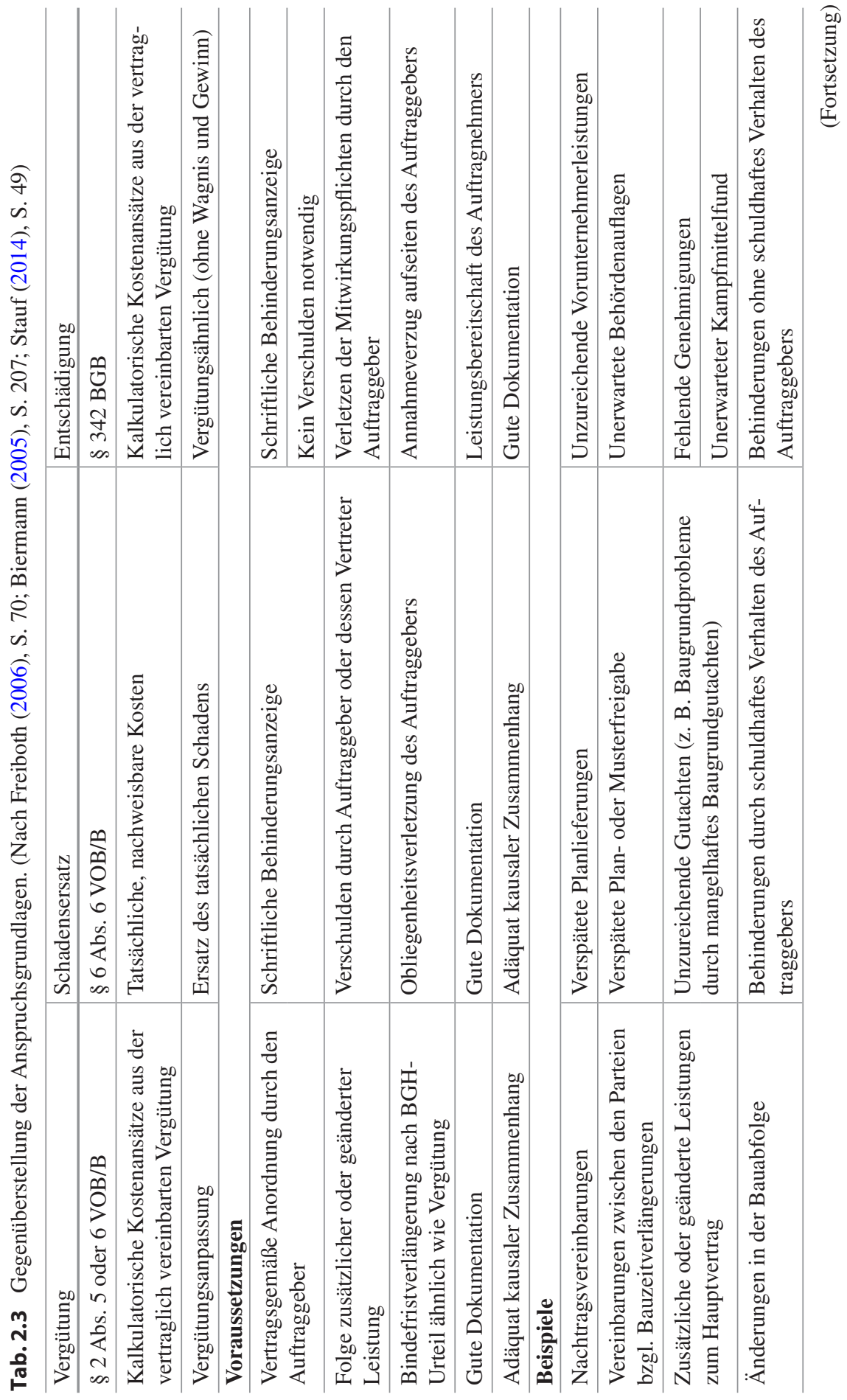




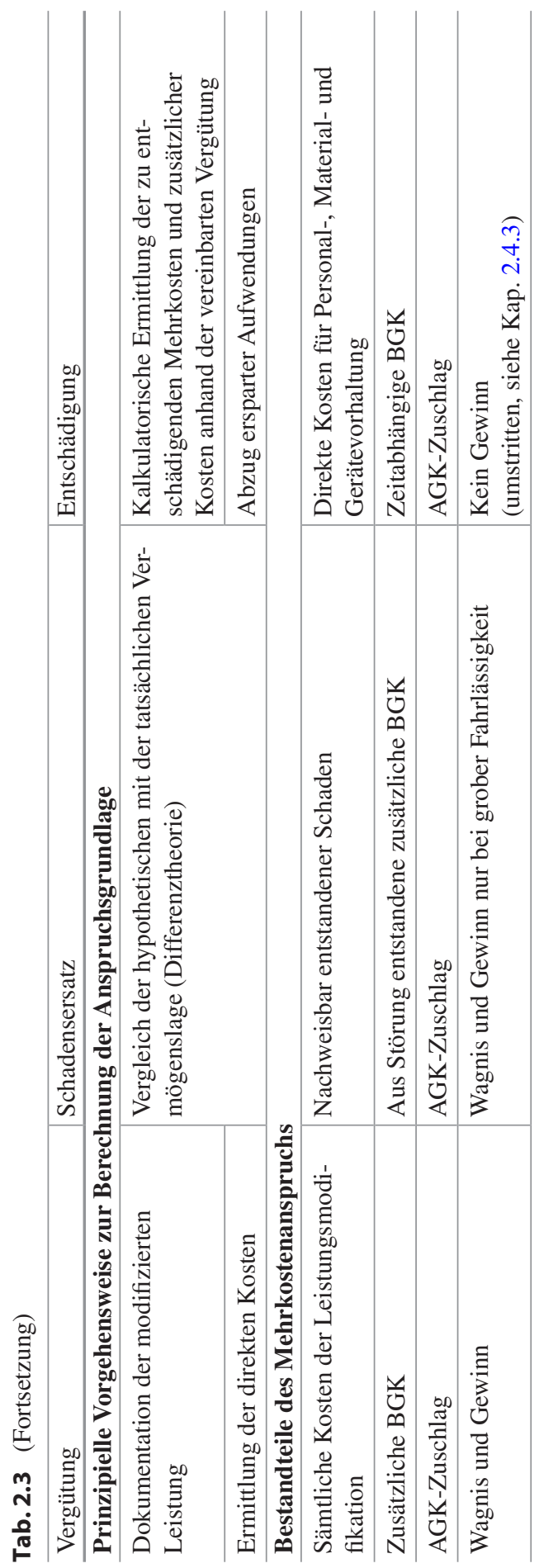


Prinzipiell treten bei der Ermittlung der zeitlichen Auswirkungen einer Störung beim Nachweis der Entschädigung dieselben Probleme auf wie bei einem Schadensersatznachweis. Das Vorliegen der Voraussetzungen der Anspruchsgrundlage ist im Vergleich zwar einfacher nachzuweisen bzw. in einigen Fällen sogar erst möglich, die Darstellung des störungsbedingt modifizierten Ablaufplanes ist aber gleichermaßen aufwendig. ${ }^{57}$

Bei der Ermittlung der Höhe des Entschädigungsanspruches entspricht die grundsätzliche Vorgehensweise der der Vergütung im Sinne der Urkalkulation. Auch hier ist eine gute Dokumentation im Bereich des ursprünglichen vereinbarten Vertragspreise, sprich der Urkalkulation, notwendig, damit das Preisniveau detailliert bestimmt werden kann.

Der Unterschied zwischen Entschädigung und Schadensersatz liegt demnach in erster Linie in der Schuldhaftigkeit des Auftraggebers. Bei Entschädigungsansprüchen kommt es nicht auf ein Verschulden des Auftraggebers an. Da die weiteren Voraussetzungen ähnlich sind und das Durchsetzen von Ansprüchen auf Entschädigung leichter erscheint, „wird ein Auftragnehmer bei Vorliegen der Voraussetzungen zukünftig seine Ansprüche nur noch auf $\S 642$ BGB stützen [...], vorausgesetzt, er erhält über die ,Entschädigung ‘ des $§ 642$ BGB dasselbe oder mehr als über den ,Schadensersatz' des $§ 6$ Nr. 6 VOB/B; nur wenn dieser Schadensersatzanspruch höher ist, wird er den Anspruch auf Verschulden stützen".58

Ansprüche im Sinne der $\S \S 6$ Abs. 6 VOB/B und 642 BGB können dem Auftragnehmer auch zusätzlich zu Vergütungsansprüchen infolge einer Anordnung zustehen, da sich die Anspruchsgrundlagen überschneiden und ergänzen können. ${ }^{59}$ Dies kann etwa von Bedeutung sein, wenn es aufgrund einer geänderten Leistung zwar zu einer Neuvereinbarung des Einheitspreises i.S. des $§ 2$ Abs. 5 VOB/B kommt, sich dadurch aber gleichzeitig eine verlängerte Bauzeit ergibt, wodurch sich Vorhaltekosten beispielsweise hinsichtlich der Baustelleneinrichtung erhöhen. Wichtig ist dennoch, dass diese Kosten direkt neben den Kosten der Vergütung geltend gemacht werden, da „Nachtragsangebote gemäß $§ 2$ VOB/B [...] alle durch die Änderungsanordnung bedingten, also verursachten Mehrkosten einschließlich der zeitabhängigen abdecken müssen, da im Falle der Beauftragung des Nachtragsangebots für den Auftragnehmer keine Möglichkeit mehr besteht, die durch Bauzeitverlängerung entstehenden oder entstandenen Mehrkosten gesondert über $\S 6$ Nr. 6 VOB/B geltend zu machen."60 In der Praxis sprechen Auftragnehmer in diesem Fall erfahrungsgemäß oft Vorbehalte aus, die ihnen eine spätere Berechnung ermöglichen soll.

\footnotetext{
${ }^{57}$ Vgl. Freiboth (2006), S. 70.

${ }^{58}$ Kapellmann und Schiffers (2006), S. 762, Rdn. 1648.

${ }^{59}$ Vgl. Vygen et al. (2002), S. 179, Rdn. 252.

${ }^{60}$ Vygen et al. (2002), S. 179, Rdn. 252.
} 


\subsection{Aktuelle Rechtsprechung zu Bauablaufstörungen}

Im nachfolgenden Kapitel werden die wichtigsten Urteile bezüglich Bauablaufstörungen dargestellt, und dann die von der Rechtsprechung gestellten Anforderungen an die Nachweisführung erläutert.

Hinweis: Bei den zitierten Urteilen der Oberlandesgerichte wurde bei der Auswahl darauf geachtet, dass diese nicht von nachfolgenden Urteilen des BGH revidiert wurden und demnach die aktuelle Rechtsprechung wiedergeben.

\subsubsection{Maßgebliche Urteile}

\subsubsection{Zur Wahl der Anspruchsgrundlage}

Ein wegweisendes Urteil zu Bauablaufstörungen hat der BGH im Jahre 1999 gefällt. Mit dem sogenannten „Vorunternehmer II“-Urteil ${ }^{61}$ wurde nicht nur bis dahin geltendes Recht revidiert, sondern auch erstmals entschieden, dass Auftragnehmer die Möglichkeit haben, Ansprüche aus dem BGB auch bei VOB-Verträgen geltend zu machen. Wörtlich heißt es hier:

- „Der Auftraggeber kann dem Nachunternehmer aus $\$ 642$ BGB haften, wenn er durch das Unterlassen einer bei der Herstellung des Werkes erforderlichen und ihm obliegenden Mitwirkungshandlung in den Verzug der Annahme kommt. "62

Bis zu diesem Zeitpunkt ${ }^{63}$ galt $\S 6$ Abs. 6 VOB/B (Schadensersatz) als alleinige Anspruchsgrundlage zur Geltendmachung von Ansprüchen aus störungsbedingten Mehrkosten für den Auftragnehmer. Bei VOB-Verträgen konnten demnach keine Entschädigungsansprüche aus dem BGB geltend gemacht werden. ${ }^{64}$

$\mathrm{Zu}$ beachten sind die weiteren Ausführungen zur Äquivalenz der beiden Anspruchsgrundlagen. Nach dem Wortlaut des $§ 642$ BGB ist keine Behinderungsanzeige erforderlich, der BGH hat jedoch entschieden, dass eine solche Anzeige Voraussetzung für die Geltendmachung von Entschädigungsansprüchen bei einem VOB-Vertrag ist. ${ }^{65}$ Der BGH hat ferner entschieden, welche Voraussetzungen eine Behinderungsanzeige erfüllen

\footnotetext{
${ }^{61}$ BGH-Urteil VII ZR 185/98 vom 21.10.1999.

${ }^{62}$ BGH-Urteil VII ZR 185/98 vom 21.10.1999

${ }^{63}$ Der BGH hat mit der Entscheidung aus dem Jahr 1999 seine frühere Rechtsprechung aus dem sog. ,Vorunternehmer I“-Urteil VII ZR 23/84 aus dem Jahre 1985 revidiert. Darin hatte der BGH entschieden: „Übernimmt der Auftraggeber keine ausdrückliche Einstandspflicht für das Handeln seiner beschäftigten Unternehmen, so unterliegt er keiner Haftung [...] gegenüber einem Nachfolgeunternehmer für Fehler des Vorunternehmers."
}

${ }^{64} \mathrm{Vgl}$. GPA (2007), S. 1.

${ }^{65}$ Vgl. Freiboth (2006), S. 24. 
muss. Demnach hat „der Auftragnehmer [...] in der Behinderungsanzeige anzugeben, ob und wann seine Arbeiten [...] nicht oder nicht wie vorgesehen ausgeführt werden können."66 Weiter müsse der Stillstand adäquat-kausal durch die hindernden Umstände verursacht sein und die Behinderungsanzeige müsse alle Tatsachen enthalten, aus der mit hinreichender Klarheit die Gründe für diese Behinderung ersichtlich seien. ${ }^{67}$

Das OLG Köln hat in einem Beschluss vom 27.10.2014 (Az.11 U 70/13) zudem festgelegt, unter welchen Voraussetzungen ein Anspruch auf Mehrvergütung durch eine Bauzeitverlängerung in Betracht kommt:

- „Eine Verlängerung der Bauzeit begründet nur bei einer Anordnung des Auftraggebers einen Anspruch des Auftragnehmers auf Mehrvergütung nach $\$ 2$ Nr. 5 VOB/B. Beruht die Verlängerung auf sonstigen Baubehinderungen, kommen Ansprüche des Auftragnehmers nur nach $\$ 6$ Nr. 6 VOB/B oder $\$ 642$ BGB in Betracht. "68

\subsubsection{Zur Darstellung und Nachweisführung}

Ein weiteres wichtiges Urteil zu gestörten Bauabläufen stammt bereits aus dem Jahre 1986 und beschäftigt sich mit der Darstellung der Mehrkosten. Im Zuge einer Schadensersatzforderung hat der BGH mit seiner Entscheidung VII ZR 286/84 festgelegt, welche Anforderungen an die Darlegung des Schadens zu stellen sind. So muss

- „der Geschädigte im Einzelnen darlegen [...], welche konkreten Mehrkosten ihm durch die Behinderung tatsächlich entstanden sind. "69

Dass dies auch für die Entschädigung gilt, hat die Rechtsprechung verschiedentlich entschieden. So formuliert bspw. das OLG Hamm: ${ }^{70}$

- „Zur Darstellung eines Verzögerungsschadens nach $\S 6$ Nr. $6 \mathrm{VOB} / \mathrm{B}$ und $\S 642$ BGB genügt die Darlegung der Verzögerung allein nicht. Vielmehr ist unumgänglich eine konkrete bauablaufbezogene Darstellung der Behinderungen und der Schadensauswirkungen auf den bauausführenden Betrieb. “

Der BGH hat seine Entscheidungen hinsichtlich der Aufbereitung der entstandenen Mehrkosten auch in jüngerer Vergangenheit bestätigt. So heißt es in einem Urteil aus dem Jahre $2002^{71}$ :

\footnotetext{
${ }^{66}$ BGH-Urteil VII ZR 185/98 vom 21.10.1999.

${ }^{67}$ Vgl. BGH-Urteil VII ZR 185/98 vom 21.10.1999.

${ }^{68}$ Beschluss 11 U 70/13 des OLG Köln vom 27.10.2014.

${ }^{69}$ BGH-Urteil VII ZR 286/84 vom 20.02.1986.

${ }^{70}$ Urteil 17 U 56/00 des OLG Hamm vom 12.02.2004.

${ }^{71}$ BGH-Urteil VII ZR 224/00 vom 21.03.2002.
} 
- „Der Auftragnehmer muß [sic] eine Behinderung, aus der er Schadensersatzansprüche ableitet, möglichst konkret darlegen. Dazu ist in der Regel [...] eine bauablaufbezogene Darstellung notwendig “.

Den gleichen Nachweis (der konkret im Einzelnen anfallenden Kostenpunkte) fordert das OLG Brandenburg in einer aktuellen Entscheidung bei der Geltendmachung eines Anspruchs auf Mehrvergütung nach $\S 2$ Abs. 6 VOB/B bzw. $§ 2$ Abs. 5 VOB/B. Zudem müsse ebenso nachgewiesen sein, dass die Störung in den Verantwortungsbereich des Auftraggebers fällt. In einem Urteil zu einer Bauzeitverlängerung aus dem Februar 2016 heißt es:

- „Der Auftragnehmer, der einen Anspruch auf Vergütung oder Erstattung von Mehrkosten wegen einer Bauzeitverlängerung geltend macht, hat im Einzelnen konkret darzulegen, dass die Mehrkosten auf einer vom Auftraggeber zu verantwortenden Bauzeitverlängerung beruhen. "72

Zudem bestätigt es weitere Voraussetzungen:

- „Verlangt der Auftragnehmer eine Entschädigung aus $\$ 642$ BGB, muss er die Verletzung einer dem Auftraggeber obliegenden Mitwirkungspflicht, den Annahmeverzug und dessen Dauer sowie die Grundlagen der Entschädigung, die aus der dem Vertrag zugrunde liegenden Vergütungsvereinbarung abzuleiten sind, darlegen und beweisen. "73

Da sich der BGH wiederholt auf den Terminus „,bauablaufbezogene Darstellung“ beruft, ist Roquette der Auffassung, dass diese auch bei einer Beschleunigung als Umkehrung der Bauzeitverlängerung vorliegen müsse, um darzulegen, wie sich Leistungen verschoben und in welchem Umfang diese sich verändert haben. ${ }^{74}$ Da eine Beschleunigung in den meisten Fällen aufgrund einer Anordnung des Auftraggebers erfolgt, folgt hieraus, dass diese Nachweisführung neben $\S 6$ Abs. 6 VOB/B und $\S 642$ BGB auch für Ansprüche aus $\S 2$ Abs. 5 bzw. Abs. 6 VOB/B gilt.

\subsubsection{Zur Ermittlung der Kosten bzw. des Schadens}

Bei der Ermittlung eines Schadens (sowohl Schadensersatz als auch Entschädigung) unterscheidet der BGH in zwei Kausalitäten. Einmal gibt es die haftungsbegründende Kausalität, die konkret bewiesen werden muss:

\footnotetext{
${ }^{72}$ Urteil 12 U 222/14 des OLG Brandenburg vom 18.02.2016.

${ }^{73}$ Urteil 12 U 222/14 des OLG Brandenburg vom 18.02.2016.

${ }^{74}$ Vgl. Roquette et al. (2013), S. 253, Rdn.841.
} 
- „Soweit die Behinderung darin besteht, daß [sic] bestimmte Arbeiten nicht oder nicht in der vorgesehenen Zeit durchgeführt werden können, ist sie nach allgemeinen Grundsätzen der Darlegungs- und Beweislast zu beurteilen. Der Auftragnehmer hat deshalb darzulegen und nach \$286 ZPO Beweis dafür zu erbringen, wie lange die konkrete Behinderung andauerte. "75

Und zum anderen die haftungsausfüllende Kausalität, die geschätzt werden darf:

- „Dagegen sind weitere Folgen der konkreten Behinderung nach §287 ZPO zu beurteilen, soweit sie nicht mehr zum Haftungsgrund gehören, sondern dem durch die Behinderung erlittenen Schaden zuzuordnen sind."76

\subsubsection{Zur Bindefristverlängerung}

Die Bindefristverlängerung stellt einen Sonderfall dar. Dies zeigt sich auch an der Vielzahl der Verfahren die hierzu durch die Instanzenzüge zum BGH geführt werden. Bereits im Jahre 2005 hat das OLG Jena geurteilt, dass alle Risiken, die durch das Vergabeverfahren entstehen, grundsätzlich beim Auftraggeber liegen. ${ }^{77}$ Dies hat der BGH im Urteil VII ZR 11/08 vom 11. Mai 2009 bestätigt. Hiernach ergibt sich für Auftragnehmer die Grundlage für eine Vergütungsanpassung in Anlehnung an die Grundsätze des $§ 2$ Abs. 5 VOB/B, wenn sich die Bauzeit durch ein Vergabenachprüfungsverfahren verschiebt.

- „Der so zustande gekommene Bauvertrag ist ergänzend dahin auszulegen, dass die Bauzeit unter Berücksichtigung der Umstände des Einzelfalls und der vertragliche Vergütungsanspruch in Anlehnung an die Grundsätze des $\S 2$ Nr. 5 VOB/B anzupassen sind. "78

Im selben Jahr wurde zudem entschieden, dass der Auftrag nur dann Ansprüche geltend machen kann, wenn sich durch das Vergabeverfahren auch der Baubeginn verschoben hat. Dies wird in Abb. 2.2 dargestellt.

- „Wird in einem Vergabeverfahren aufgrund öffentlicher Ausschreibung nach VOB/A der Zuschlag nach Verlängerung der Bindefristen durch die Bieter später erteilt als in der Ausschreibung vorgesehen, kann ein Mehrvergütungsanspruch nicht allein daraus hergeleitet werden, dass sich im Hinblick auf die spätere Zuschlagserteilung die Kalkulationsgrundlagen geändert haben. “79

\footnotetext{
${ }^{75}$ BGH-Urteil VII ZR 225/03 vom 24.02.2005.

${ }^{76}$ BGH-Urteil VII ZR 225/03 vom 24.02.2005

${ }^{77} \mathrm{Vgl}$. Urteil 8 U 318/04 des OLG Jena vom 22.03.2005.

${ }^{78}$ BGH-Urteil VII ZR 11/08 vom 11.05.2009.

${ }^{79}$ BGH-Urteil VII ZR 82/08 vom 10.09.2009.
} 


\section{Normaler Ablauf:}
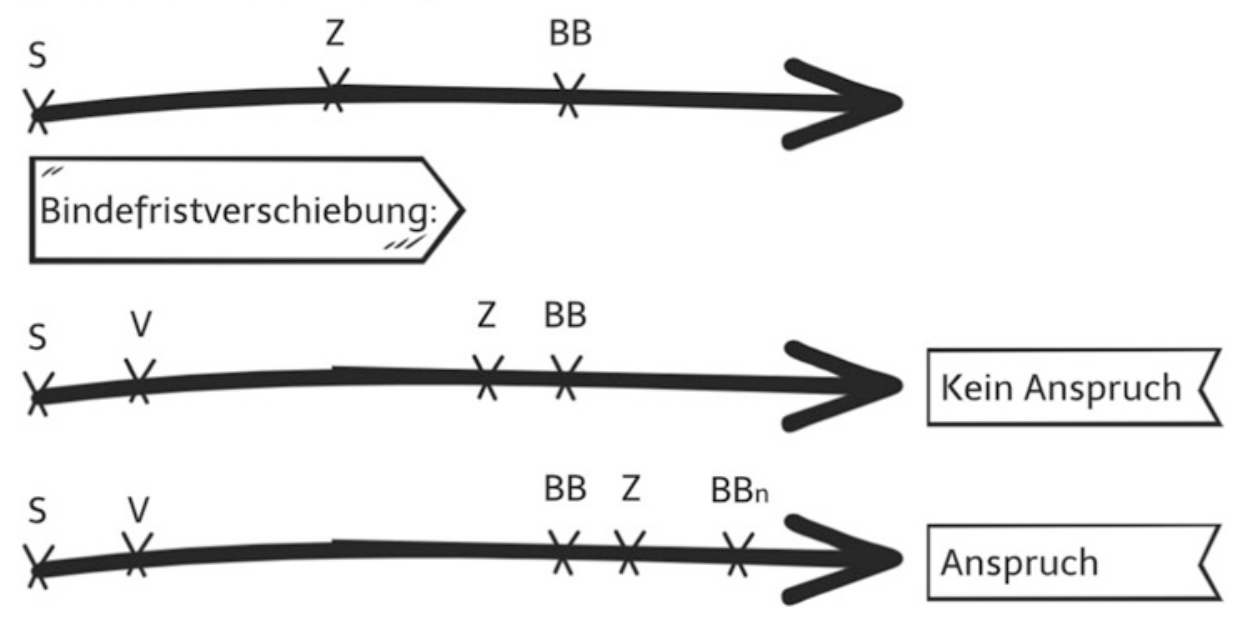

Abb. 2.2 Anspruch bei Bindefristverlängerung. (In Abb. 2.2 beschreibt „S“ den Zeitpunkt der Submission, „Z“ den Zuschlagstermin, „BB“ den geplanten Baubeginn, „V“ den Vergabeeinspruch und ,BB ${ }_{\mathrm{n}}$ " den neuen Baubeginn)

Sobald sich die Ausführungszeit und damit die Leistungspflichten verändern, können alle Mehrkosten geltend gemacht werden, die ursächlich auf die Verschiebung der Bauzeit zurückzuführen sind. Dies hat der BGH ebenfalls in einem Urteil aus dem Jahr 2009 entschieden.

- „Maßgeblich für die Ermittlung der Höhe der an die Klägerin zu zahlenden Mehrvergütung sind diejenigen Mehrkosten, die ursächlich auf die Verschiebung der Bauzeit zurückzuführen sind. Sie ergeben sich im rechtlichen Ausgangspunkt aus der Differenz zwischen den Kosten, die bei der Klägerin für die Ausführung der Bauleistung tatsächlich angefallen sind und den Kosten, die sie bei Erbringung der Bauleistung in dem nach der Ausschreibung vorgesehenen Zeitraum hätte aufwenden müssen. [...] Das Landgericht wird in Anwendung des obigen Grundsatzes den tatsächlich angefallenen Einkaufspreisen also diejenigen Preise gegenüberstellen, welche die Klägerin bei Einhaltung der ursprünglich vorgesehenen Bauzeit hätte zahlen müssen. "80

Diese Ausführungen bestätigt der BGH auch im Jahre 2012 mit dem Urteil VII ZR 202/09. Hier wird zudem entschieden, dass auch gestiegene Kosten im Hinblick auf Nachunternehmerverträge geltend gemacht werden können.

\footnotetext{
${ }^{80}$ BGH-Urteil VII ZR 152/08 vom 10.09.2009.
} 
- „Bei Anwendung dieser Grundsätze kann einem Auftragnehmer ein Mehrvergütungsanspruch in Höhe des Betrages zustehen, der sich aus der Differenz zwischen den tatsächlich durch die Beauftragung eines Nachunternehmers entstandenen Kosten und denjenigen Kosten ergibt, die für ihn bei Einhaltung der ursprünglichen Bauzeit durch die Annahme des bindenden Angebots eines günstigeren Nachunternehmers entstanden wären. “81

\subsubsection{Mindestanforderungen an die Nachweisführung}

Bislang $^{82}$ gibt es keine höchstrichterliche Entscheidung über die Mindestanforderungen an die Nachweisführung bei der Durchsetzung von Ansprüchen aus Bauablaufstörungen, wodurch es zwischen den Beteiligten oft zu Streitigkeiten kommt. Dies betrifft vor allem die Fälle des Schadensersatzes und der Entschädigung, da die zusätzliche Vergütung eine Anordnung voraussetzt, wodurch es hier weniger Streitpotenzial gibt. Sobald allerdings die Bauzeit betroffen ist, oder es im Falle der Bindefristverlängerung zu Verschiebungen kommt, kann es auch hier zu Streitigkeiten kommen.

So ist beispielsweise auch Viering der Ansicht, dass die ,Durchsetzung bauzeitbedingter Mehrkosten oftmals [...] schwierig [und] die Erfolgsaussichten bei der gerichtlichen Durchsetzung [...] schwer vorhersehbar und oftmals gering ${ }^{\text {"83 }}$ sind.

Dennoch ist es unvermeidbar, sich dieser Situation im Baualltag zu stellen. Auch wenn die Anforderungen der Rechtsprechung nur von einem Bauleiter mit technischem, kaufmännischem und juristischem Wissen bearbeitet werden könne, der zudem über eine hohe Qualifikation und Zeit verfüge ${ }^{84}$, sei es die Aufgabe des Bauleiters einen gerichtsfesten Kostennachweis aufzustellen, wodurch diese allerdings oftmals überfordert $\operatorname{seien}^{85}$.

In einem Urteil VII ZR 224/00 vom 21.03.2002 hat der BGH entschieden, dass der entstandene Schaden möglichst konkret dargestellt werden müsse. An anderen Stellen heißt es die Nachweisführung solle ,,adäquat kausal“ ${ }^{66}$ und „,möglichst konkret [und] bauablaufbezogen" 87 dargestellt werden. Konkretisiert hat der BGH seine Rechtsprechung im Jahr 2005 im Urteil VII ZR 141/03. In dem heißt es, dass der

\footnotetext{
${ }^{81}$ BGH-Urteil VII ZR 202/09 vom 08.03.2012.

${ }^{82}$ Stand: Frühjahr 2020.

${ }^{83}$ Zanner et al. (2014), S. 57.

${ }^{84} \mathrm{Vgl}$. Heilfort (2003), S. 458.

${ }^{85} \mathrm{Vgl}$. Mitschein (1999), S. 7.

${ }^{86}$ BGH-Urteil VII ZR 224/00 vom 21.03.2002.

${ }^{87}$ BGH-Urteil VII ZR 224/00 vom 21.03.2002.
} 
Auftragnehmer widerspruchsfreie Angaben dazu machen müsse, aufgrund welcher Planverzögerungen welche vorgesehenen Arbeiten nicht durchgeführt werden konnten und wie sich die Planverzögerungen konkret auf die Baustelle ausgewirkt haben.

Folglich ist es nicht möglich, seine Ansprüche auf abstrakte Werte zu stützen. Vielmehr muss jederzeit der Bezug zum tatsächlichen Ist-Zustand auf der Baustelle hergestellt werden, was nur mithilfe einer organisierten Dokumentation aller Abläufe geschehen kann. Dieser erste Schritt der ausführlichen Dokumentation ist erforderlich, um den Anspruch dem Grunde nach nachzuweisen ${ }^{88}$ und dient außerdem dazu, dass der Anspruch vom Auftraggeber nicht als nicht prüfbar zurückgewiesen werden kann. Erst in einem zweiten Schritt wird die Höhe des Anspruches betrachtet.

Bei der Beurteilung von Ansprüchen aus Bauablaufstörungen, unabhängig von der gewählten Anspruchsgrundlage, müssen die Mehrkosten und deren Ursache detailliert dargelegt werden.

Um ein Scheitern der Nachtragsforderung zu vermeiden, ist es erforderlich, die Forderung nachvollziehbar und verständlich aufzubauen. Unmittelbar am Projekt Beteiligte berücksichtigen oftmals nicht, dass Außenstehenden, die sich mit der Forderung auseinandersetzen müssen, das Projekt nicht so detailliert bekannt ist, wie für die Personen, die das Projekt von Beginn an betreuen. ${ }^{89}$

Als richtungsweisend zum Thema der Nachweisführung gilt neben dem oben genannten Urteil aus dem Jahre 2002 auch das Urteil VII ZR 225/03 vom 24.02.2005, in welchem der BGH erneut von einer ,konkreten, bauablaufbezogenen Darstellung der jeweiligen Behinderung“" spricht. Weiter heißt es, dass der Auftragnehmer dazulegen hat, „wie lange die konkrete Behinderung“ andauere und welche Folgen diese nach sich ziehe.

Aus beiden Urteilen ist deutlich zu entnehmen, dass die Darlegungs- und Beweislast für die Störung und deren Folgen allein beim Auftragnehmer liegt. Obwohl es in diesen konkreten Fällen um einen Schadensersatzanspruch gemäß $§ 6$ Abs. 6 VOB/B ging, kann das Ergebnis auf alle Anspruchsgrundlagen übertragen werden. ${ }^{90}$ „Weite Teile der Praxis glauben, diesen Nachweis [...] zwar bei Schadensersatzansprüchen, nicht aber bei Vergütungsansprüchen führen zu müssen. Dabei besteht der Schwerpunkt einer jeden Dokumentation von Bauablaufänderungen und -störungen weniger in der Darlegung der unterschiedlichen Anspruchsvoraussetzungen [...]. Der Schwerpunkt liegt vielmehr in dem allen Ansprüchen gemeinsamen, plausiblen und schlüssigen Kausalitätsnachweis." "91

\footnotetext{
${ }^{88}$ Vgl. Dreier (2001), S. 2.

${ }^{89}$ Vgl. Dreier (2001), S. 3.

${ }^{90} \mathrm{Vgl}$. Zanner et al. (2014), S. $63 \mathrm{f}$.

${ }^{91}$ Tomic (2014), S. 348, Rdn. 6.
} 


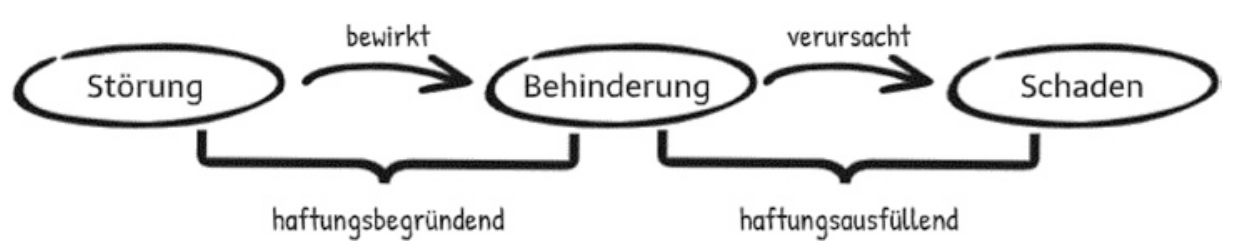

Abb. 2.3 Haftungsbegründende und haftungsausfüllende Kausalität. (Nach Leitzke 2006, S. 131)

\subsubsection{Der Kausalitätsnachweis}

Der $\mathrm{BGH}^{92}$ unterscheidet in der Umsetzung der Nachweisführung generell in die haftungsbegründende und die haftungsausfüllende Kausalität gemäß §§ 286 f. ZPO. Dies ist in Abb. 2.3 näher erläutert.

Die haftungsbegründende Kausalität thematisiert allein die Haftung, also die Frage, ob ein Schädiger eine bestimmte Störung verursacht hat ${ }^{93}$. Bei der haftungsausfüllenden Kausalität geht es darüber hinaus um „den konkreten Vortrag bzgl. des Zusammenhanges zwischen Behinderung und baubetriebswirtschaftlichen Folgen“"94, also die Höhe des Anspruchs. Hierbei kommt „für den Auftragnehmer zum Tragen, sofern die haftungsbegründende Kausalität erfüllt ist, dass er bei einer gerichtlichen Auseinandersetzung eine Schadensschätzung gemäß § 287 ZPO durch das Gericht beanspruchen kann, wenn er ausreichend Grundlagen für die Schadensschätzung darlegt. “95 Wotschke hat demzufolge erkannt, dass eine solche Schätzung nicht mit einem Auswürfeln zu verwechseln sei und es auch eine nachvollziehbare Bewertungsgrundlage in Form von sauberer Dokumentation brauche. ${ }^{96}$ Welche Bestandteile zu einer solchen Dokumentation gehören, wird in Abschn. 2.5.2.3 näher erläutert.

Nach der herrschenden Meinung der Literatur und der Rechtsprechung ist demnach die haftungsbegründende Kausalität zunächst sehr viel wichtiger und nicht zu vernachlässigen, wenn Ansprüche aus Bauablaufstörungen erfolgreich durchgesetzt werden sollen.

Zunächst ist zu erläutern, was unter der vom BGH geforderten adäquaten Kausalität zu verstehen ist. Für Baupraktiker bedeutet dies auf den Punkt gebracht:

1. Die Ursache und deren Wirkung muss dargestellt werden. (von lat. causa - Ursache)

2. Es muss der Bezug zwischen der Handlung eines Schädigers und dem Schaden erkennbar sein. (von lat. adaequare - gleichmachen/angemessen)

\footnotetext{
${ }^{92} \mathrm{Vgl} \mathrm{u.} \mathrm{a.} \mathrm{BGH-Urteil} \mathrm{VII} \mathrm{ZR} \mathrm{141/03} \mathrm{vom} \mathrm{24.02.2005.}$

${ }^{93}$ Vgl. Zimmermann J. (2010), S. 78.

${ }^{94}$ Schottke (2006), S. 8.

${ }^{95}$ Schmitt S. (2006), S. 101.

${ }^{96}$ Vgl. Wotschke und Wotschke (2006), S. 63.
} 
Tab. 2.4 Nachweispflichten des Auftragnehmers. (Nach Zanner et al. 2014, S. 70-71)

\begin{tabular}{|c|c|}
\hline Vollbeweispflichtig ${ }^{\mathrm{a}}$ & Beispiel \\
\hline Störung & $\begin{array}{l}\text { Planübergabe durch den Auftraggeber verspätet sich um } \\
4 \text { Wochen }\end{array}$ \\
\hline Die daraus resultierende Behinderung & Die Betonage der 1. Sohle verspätet sich um 4 Wochen \\
\hline Die Dauer der Behinderung & 4 Wochen \\
\hline Der Kausalzusammenhang & $\begin{array}{l}\text { Der verspätete Beginn der Betonage ist auf die } \\
\text { fehlenden Pläne zurückzuführen und nicht bspw. auf } \\
\text { schlechte Witterung }\end{array}$ \\
\hline \multicolumn{2}{|l|}{ Nicht vollbeweispflichtig ${ }^{\mathrm{b}}$} \\
\hline Auswirkungen auf weitere Termine & $\begin{array}{l}\text { Der Gesamtfertigstellungstermin verzögert sich um } 4 \\
\text { Wochen }\end{array}$ \\
\hline Der resultierende Mehraufwand & $\begin{array}{l}4 \text { Wochen längere/r Personaleinsatz, Vorhaltung von } \\
\text { Geräten etc. }\end{array}$ \\
\hline
\end{tabular}

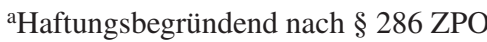

${ }^{b}$ Haftungsausfüllend - weitere Behinderungsfolgen auf den Bauablauf (dürfen geschätzt werden)

Bei einer Übertragung auf den gestörten Bauablauf liegt ein adäquater Kausalzusammenhang dann vor, wenn durch eine Störung, z. B. durch eine aktive Anordnung des Auftraggebers oder auch durch sogenanntes Unterlassen z. B. durch eine verspätete oder fehlende Planübergabe, Verzögerungen im Bauablauf entstehen.

Es reicht dabei nicht aus, die gestörten Bauabläufe abstrakt und allgemein darzulegen. Der BGH hat mehrfach dargestellt, dass die sich die Darstellung im Detail mit den Einzelheiten der Störung und deren Auswirkungen auf den Ablauf beschäftigen müsse ${ }^{97}$.

Die ZPO unterscheidet in der Beweisführung dabei in vollbeweispflichtige und nicht vollbeweispflichtige Bereiche, die in Tab. 2.4 dargestellt sind. Hier wird außerdem aufgezeigt, was der Auftragnehmer für einen ausreichenden Kausalitätsnachweis beweisen muss.

Für die praktische Umsetzung bedeutet dies, dass zunächst das störende Ereignis im Einzelnen, und anschließend auch die dadurch entstandene Verzögerung dargestellt werden muss. Es ist also erforderlich, genau darzulegen, welche Leistungen zum Zeitpunkt der Störung ausgeführt werden sollten und nun nicht bzw. nur unter Behinderung ausgeführt werden können. Hinzu kommen die Auswirkungen der einzelnen Störung auf den gesamten Bauablauf, die konkretisiert und belegt werden müssen. ${ }^{98}$

\footnotetext{
${ }^{97}$ Vgl. Mechning et al. (2014), S. 85.

${ }^{98} \mathrm{Vgl}$. Roquette et al. (2013), S. 169, Rdn 542 f.
} 
Eine gute Methodik zur Visualisierung dieser geforderten bauablaufbezogenen Darstellung ist die sogenannte Soll'-Methode ${ }^{99}$, die auch der BGH als auskömmlich erkennt. ${ }^{100}$

\subsubsection{Die bauablaufbezogene Darstellung mithilfe der Soll'-Methode}

Die Soll'-Methode läuft in drei Schritten ab. In einem ersten Schritt wird der sogenannte Soll-Ablauf modellhaft ${ }^{101}$ bestimmt. Dieser zeigt den planmäßigen Ablauf des gesamten Bauvorhabens detailliert bis zur Fertigstellung und bildet alle vertraglichen Termine und Rahmenbedingungen ab. Dieser Soll-Ablaufplan, auch Null-Plan genannt, ist nachfolgend die Basis für die weiteren Schritte und im Idealfall bereits als ,Vertragsterminplan“ Vertragsbestandteil. Unter anderem Bötzkes ${ }^{102}$ empfiehlt, dass den Vorgängen direkt Kapazitäten (wie z. B. Kolonnenstärken und -anzahl) hinterlegt werden sollten. Dies hätte den Vorteil, dass, sobald der Soll-Ablaufplan vertraglich vereinbart sei, der Auftragnehmer auch bei einem gestörten Bauablauf nicht mehr als die ursprünglich geplanten Kapazitäten einsetzen müsse. Sollte der Soll-Ablaufplan nicht vertraglich vereinbart sein, ist er prinzipiell auch nachträglich erstellbar. ${ }^{103}$ Das OLG Brandenburg geht sogar so weit, dass es Vergütungs- und Schadensersatzansprüche (auch bei Entschädigung) wegen Bauablaufstörungen ausschließt, sollte kein verbindlicher Bauzeitenplan vereinbart sein. ${ }^{104}$

Sollten sich Leistungsänderungen oder -ergänzungen i.S. der $\S \S 2$ Abs. 5 und Abs. $6 \mathrm{VOB} / \mathrm{B}$ oder Mehr- und Minderleistungen gemäß $\S 2$ Abs. 3 VOB/B, also vertragskonforme Modifikationen ergeben, müssen diese mit in den Soll-Ablaufplan eingearbeitet werden. Dies hat zunächst nichts mit einer Störung zu tun, sondern dient ausschließlich zur Feststellung der Ausgangssituation.

Im zweiten Schritt muss die Störung festgestellt, dokumentiert und analysiert werden. Dafür werden in einem Soll-Ist-Vergleich Abweichungen mithilfe baustellenbezogener Dokumentation (siehe Abschn. 2.5.2.3) festgestellt.

In einem dritten und letzten Schritt kann dann der Soll'-Ablaufplan, auch als störungsbedingt modifizierter Ablaufplan bezeichnet, erstellt werden. Hier wird die

\footnotetext{
${ }^{99}$ Gesprochen: Soll-Strich-Methode - es gibt sowohl den Soll-Ablauf, als auch den Soll-StrichAblauf.

${ }^{100}$ Siehe BGH-Urteil VII ZR 286/84: „Ausgangspunkt [...] ist der vom Auftragnehmer in seiner Kalkulation zugrunde gelegte Bauabablauf (Soll). Dem wird ein sogenannter störungsmodifizierter Bauablauf gegenübergestellt [Soll'].“

${ }^{101}$ Roquette et al. (2013) merken richtigerweise an, dass die Darstellung konkret und bauablaufbezogen sein muss, dies aber baupraktisch kaum möglich ist und eine Darstellung daher bis zu einem gewissen Maße nur modellhaft erfolgen kann (siehe Rdn. 551).

${ }^{102}$ Bötzkes (2010), S. $146 \mathrm{f}$.

${ }^{103}$ Hierzu sei auf das BGH-Urteil VII ZR 201/06 vom 18.12.2008 verwiesen, in welchem der BGH die nachträgliche Vorlage der Urkalkulation zugelassen hat. Es ist nicht ersichtlich, warum dies nicht auch gleichermaßen für einen Bauzeitenplan gelten soll.

${ }^{104}$ Vgl. Urteil 11 U 102/12 des OLG Brandenburg vom 02.12.2015.
} 
Störung in den ursprünglichen Plan eingearbeitet, wodurch die konkreten Auswirkungen der Störungen nachvollzogen werden können. Die „Auswirkung [muss] so beschrieben werden, dass sie dem tatsächlichen ,Bau-Ist" möglichst entspricht“" ${ }^{105}$ Dies beinhaltet auch zusätzliche und geänderte Leistungen, sowie Minderleistungen und Zuschläge für die Wiederaufnahme und andere Produktivitätsverluste, die einbezogen werden müssen.

\subsubsection{Nachweis der Verantwortung aufseiten des Auftraggebers durch eine richtige Dokumentation}

Ein ausreichender Nachweis über das Verschulden des Auftraggebers, wie er in Schadensersatzfällen nötig ist, kann dem Auftragnehmer nur dann gelingen, wenn er eine detaillierte Dokumentation über die Baustelle führt. Ist dies nicht der Fall, kann ihm einerseits Organisationsverschulden vorgeworfen werden und andererseits hat er ohne eine sorgfältige Baustellendokumentation kaum Möglichkeiten, seine Ansprüche gegenüber dem Auftraggeber durchzusetzen. ${ }^{106}$

Da die juristischen Anforderungen im Schadensersatzfall für den Bauunternehmer ein beinahe utopisches Ausmaß erreichen (siehe Abschn. 2.4.4) wird der Auftragnehmer bevorzugt die Entschädigung als Anspruchsgrundlage wählen. Die Verantwortung aufseiten des Auftraggebers muss allerdings auch in diesem Fall nachgewiesen werden, auch wenn er nicht direkt schuldhaft beteiligt sein muss. Eine Verantwortung nachzuweisen ist zwar einfacher als eine Schuld nachzuweisen, dennoch ist auch hier eine detaillierte Dokumentation notwendig, wenn Ansprüche erfolgreich durchgesetzt werden sollen.

Tomic beschreibt die Dokumentation als wichtigsten Faktor für eine erfolgreiche Nachtragsforderung. Sie sei als obligate Voraussetzung und nicht als mühsame Verwaltungsarbeit zu sehen, die vor allem als Grundlage für die Durchsetzung dem Grunde nach diene und ablaufbegleitend und nicht nachträglich zu erstellen sei. ${ }^{107}$

Wichtig ist weiterhin, dass für jede Störung eine konkrete, einzelfallbezogene und umfangreiche Darstellung zu den Umständen vorgetragen werden kann. Der Auftragnehmer hat mithilfe der Dokumentation darzulegen, welche Umstände aus dem Bereich des Auftraggebers wie und zu welchem Teil von Störungen bzw. daraus resultierenden Bauzeitverlängerungen führen. ${ }^{108}$

Tab. 2.5 zeigt auf, welche Bestandteile eine sorgfältige Dokumentation beinhalten sollte. $\mathrm{Zu}$ dieser ist der Auftragnehmer im Übrigen selbst bei nicht gestörten Bauabläufen verpflichtet. ${ }^{109}$

\footnotetext{
${ }^{105}$ Vgl. Roquette et al. (2013) S. 201, Rdn. 615.

${ }^{106} \mathrm{Vgl}$. IWW (2008), S. 14.

${ }^{107} \mathrm{Vgl}$. Tomic (2014), S. 347, Rdn. 1 f.

${ }^{108}$ Vgl. Tomic (2014), S. 361, Rdn. 45.

${ }^{109}$ Nach dem BGH-Urteil VII ZR 5/91 vom 12.03.1992 muss der Auftragnehmer für eine angemessene Überwachung und Prüfung der Leistungen sorgen.
} 
Tab. 2.5 Dokumentation im Bauwesen. (Ergänzt nach Mitschein (1999), S. 62 ff.; Reister (2014), S. 462 ff., ohne Anspruch auf Vollständigkeit)

\begin{tabular}{|c|c|}
\hline Bautagebuch & $\begin{array}{l}\text { Beinhaltet: Kapazitäten (Arbeitskräfte, Führungspersonal, Geräte, } \\
\text { etc.), äußere Bedingungen, besondere Vorkommnisse, Anordnungen, } \\
\text { usw. } \\
\text { Evtl. gesondert: Betoniertagebuch }\end{array}$ \\
\hline Soll-/Ist-Vergleich & $\begin{array}{l}\text { Gegenüberstellung von Soll- und Ist-Stunden bei ungestörten } \\
\text { Abschnitten und bei gestörten Abschnitten zum Nachweis des Mehr- } \\
\text { verbrauches }\end{array}$ \\
\hline $\begin{array}{l}\text { Planeingangslisten } \\
\text { (Planlisten) }\end{array}$ & $\begin{array}{l}\text { Besonders wichtig, um unterlassene Mitwirkungspflichten durchzu- } \\
\text { setzen. Dienen dem stetigen Vergleich zwischen Ausführungs- und } \\
\text { Angebotsplänen. } \\
\text { Wann erfolgte die Freigabe zu welchem Plan und mit welchem } \\
\text { Index? }\end{array}$ \\
\hline Besprechungsprotokolle & $\begin{array}{l}\text { Sollten den aktuellen Leistungsstand, Behinderungen, Unter- } \\
\text { brechungen etc. beinhalten. Baubesprechungen sollten regelmäßig } \\
\text { durchgeführt werden und die zugehörigen Protokolle von beiden } \\
\text { Parteien unterzeichnet sein }\end{array}$ \\
\hline Aktennotizen & $\begin{array}{l}\text { Dienen zur Offenlegung von Änderungen in vertraglichen } \\
\text { Leistungen, enthalten Vermerke zu Planänderungen, Zusammen- } \\
\text { fassungen von Besprechungen, etc. und werden der Gegenseite } \\
\text { postalisch zugestellt }\end{array}$ \\
\hline Schriftverkehr & $\begin{array}{l}\text { Jeglicher Austausch zwischen den Parteien kann im Streitfall heran- } \\
\text { gezogen werden und wichtig sein. Beinhaltet auch Behinderungs- } \\
\text { anzeigen, Mehrkosten- \& Bedenkenanmeldungen etc. }\end{array}$ \\
\hline Fotodokumentation & $\begin{array}{l}\text { Auch später nicht mehr erkennbare Zwischenstände können auf- } \\
\text { genommen werden, Erschwernisse werden festgehalten und } \\
\text { Fertigungsabschnitte werden sichtbar }\end{array}$ \\
\hline Aufmaßprotokolle & $\begin{array}{l}\text { Dienen der Leistungsfeststellung und dem Vergleich mit den Mengen } \\
\text { des Leistungsverzeichnisses und sollten von beiden Parteien gegen- } \\
\text { gezeichnet werden }\end{array}$ \\
\hline Kalkulationsgrundlagen & $\begin{array}{l}\text { Werden für den Bezug zur Vertragsgrundlage benötigt (Mittellohn, } \\
\text { Ansätze für AGK-, BGK-, \& WuG-Zuschlag, etc.) }\end{array}$ \\
\hline
\end{tabular}

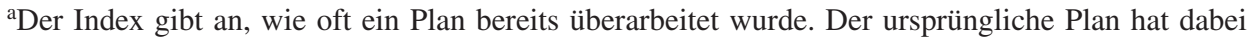
keinen Index, der erste überarbeitete Plan startet mit dem Index ,a“. Wird dieser Plan erneut überarbeitet folgt „," usw.

Hinzu kommt, dass, um eine lückenlose und vollständige Dokumentation vorzulegen, auch die Vorgeschichte der Störung aufgezeigt werden muss. Wie konnte es zu der Störung kommen? Wann ist die Störung erstmals aufgetreten? Wie wird die Ausführung dadurch beeinträchtigt? Welche Abschnitte bzw. Leistungen sind betroffen? Dies muss auch für unbeteiligte Dritte im Einzelvortrag nachvollziehbar und verständlich gemacht werden. 


\subsection{Zwischenfazit}

Bei der Selektion der Anspruchsgrundlage kann der Auftragnehmer zwischen den Anspruchsgrundlagen auswählen, vorausgesetzt die einzelnen Voraussetzungen liegen vor. Zusammenfassend lässt sich festhalten, dass Ansprüche die aufgrund einer Anordnung geltend gemacht, und damit gemäß $\S 2$ VOB/B berechnet werden, die größte Aussicht auf Erfolg haben. Sie lassen sich nach relativ klaren Vorgaben als Fortschreibung der Urkalkulation aufstellen und beinhalten somit alle Kostenpunkte.

In den anderen Fällen, der Entschädigung und dem Schadensersatz, ist die Dokumentation des Auftragnehmers der Schlüsselpunkt zu einer erfolgreichen Durchsetzung der Ansprüche bzw. zu einer Nachtragsbeauftragung durch den Auftraggeber. Es obliegt ihm nachzuweisen, ob der Auftraggeber die Ablaufstörung verschuldet oder diese zumindest zu vertreten hat. Da die Berechnung der Mehrkosten für eine Entschädigung genau wie die aufgrund einer Anordnung auf Grundlage der Urkalkulation berechnet werden kann, empfiehlt sich diese klar gegenüber der Schadensersatzforderung. Eine Schadenersatzforderung beruht auf konkret entstandenen Kosten, die entsprechend nachgewiesen werden müssen. An diesen Nachweis werden durch die Rechtsprechung sehr hohe Ansprüche gestellt.

Als logischer Konsequenz daraus folgt, dass es eines Ablaufschemas in Unternehmen bedarf, um zukünftig möglichst große Erfolgschancen beim Umgang mit Bauablaufstörungen zu haben.

\section{Literatur}

\section{Gesetze, Verordnungen, Vorschriften und Normen}

BGB (2020) Bürgerliches Gesetzbuch (BGB), Ausgabe 2020, 85. Auflage, Beck-Texte im dtv (Deutscher Taschenbuch Verlag), München

VOB (2019) Bundesvereinigung Mittelständischer Bauunternehmen e. V. (BVMB), Vergabe- und Vertragsordnung - Ausgabe 2019, Ernst Vögel Verlag, Stamsried

\section{Monographien und Beitragswerke}

Biermann M. (2005) Der Bauleiter im Bauunternehmen - Bauablaufstörungen, Nachträge, Dokumentation, 3. Aufl., Verlagsgesellschaft Rudolf Müller, Köln.

Born B.-L. (1980) Systematische Erfassung und Bewertung der durch Störungen im Bauablauf verursachten Kosten; Werner Verlag, Düsseldorf

Dreier F. (2001) Nachtragsmanagement für gestörte Bauabläufe aus baubetrieblicher Sicht, Dissertation, Universität Cottbus

Fischer P., Schonebeck K.-H., Keil W. (2001) Rechtsfragen im Baubetrieb, 4. Aufl., Werner Verlag, Düsseldorf

Freiboth A. (2006) Ermittlung der Entschädigung bei Bauablaufstörungen, Dissertation, Universität Braunschweig 
Heiermann W., Riedl R., Rusam M. (2011) Handkommentar zur VOB, Teile A und B, 12. Aufl., Vieweg + Teubner Verlag, Wiesbaden

Ingenstau H., Korbion H. (2010) VOB Teile A und B - Kommentar, 17. Aufl., Werner Verlag, Düsseldorf

Kapellmann K.-H., Schiffers K. D. (2006) Vergütung, Nachträge und Behinderungsfolgen beim Bauvertrag, Band 1: Einheitspreisvertrag, 5. völlig neu bearbeitete und erweiterte Aufl., Werner Verlag, Düsseldorf

Leineweber A. (2002) Mehrkostenforderungen des Auftragnehmers bei gestörtem Bauablauf. In: Kapellmann K. D., Vygen K. (Hrsg.) Jahrbuch Bauchrecht 2002 - Aktuelles, Grundsätzliches, Zukünftiges, Werner Verlag, Düsseldorf, S. 107-141

Mitschein A. (1999) Die baubetriebliche Bewertung gestörter Bauabläufe aus Sicht des Auftragnehmers, Dissertation, Universität Aachen

Reister D. (2014) Nachträge beim Bauvertrag, 3. Aufl., Werner Verlag, Köln

Roquette A. J., Viering M. G., Leupertz S. (2013) Handbuch Bauzeit, 2. Aufl., Werner Verlag, Düsseldorf

Schmitt S. (2006) Allgemeine Geschäftskosten, Wagnis und gewinn bei Nachträgen und Störungen; In: Tagungsbericht Nr. 8 der Interdisziplinären Tagung für Betriebswirtschaft und Baurecht - Störungen im Bauablauf, Rechtsprechungsübersicht, Nachträge und Nachtragskalkulation, 1. Aufl., SEMINA Verlag, Neustadt, S. 86-104

Schottke R. (2006) Varianten der Schätzung gemäß § 287 ZPO bei der haftungsausfüllenden Kausalität; In: Tagungsbericht Nr. 8 der Interdisziplinären Tagung für Betriebswirtschaft und Baurecht - Störungen im Bauablauf, Rechtsprechungsübersicht, Nachträge und Nachtragskalkulation, 1. Aufl., SEMINA Verlag, Neustadt, S. 4-28

Stauf D. (2014) Gestörter Bauablauf - juristische Grundlagen für bauzeitliche Ansprüche, Seminarunterlage der Bundesvereinigung Mittelständischer Bauunternehmen e. V. (BVMB) vom 04.02.2015, Hannover

Tomic A. (2014) Bauzeit und zeitabhängige Kosten - in Vergabe, Vertrag und Nachtrag, 1. Aufl., Bundesanzeiger Verlag, Köln

Vygen K., Schubert E., Lang A. (2002) Bauverzögerung und Leistungsänderung: Rechtliche und baubetriebliche Probleme und ihre Lösungen, 4. neu bearbeitete und erweiterte Aufl., Werner Verlag, Düsseldorf

Wotschke M., Wotschke P. (2006) Minderleistung durch gestörten Bauablauf - Kennwerte in Theorie und Praxis. In: Tagungsbericht Nr. 8 der Interdisziplinären Tagung für Betriebswirtschaft und Baurecht - Störungen im Bauablauf, Rechtsprechungsübersicht, Nachträge und Nachtragskalkulation, 1. Aufl., SEMINA Verlag, Neustadt, S. 62-73

Zanner R., Saalbach B., Viering M. (2014) Rechte aus gestörtem Bauablauf nach Ansprüchen Entscheidungshilfen für Auftraggeber, Auftragnehmer und Projektsteuerer, 1. Aufl., Springer Vieweg, Wiesbaden

Zimmermann J. (2010) Prozessorientierter Nachweis der Kausalität zwischen Ursache und Wirkung bei Bauablaufstörungen, Abschlussbericht, Fraunhofer IRB Verlag, Stuttgart

\section{Zeitschriften und Zeitungen}

Bötzkes F.-A. (2010) Gestörter Bauablauf - Baubetriebliche Ermittlung von Bauzeitverlängerungen und Berechnung der Mehrkosten. In: Bautechnik (Sonderdruck), 03/2010, S. 145-157

GPA (2007): o.V., Gemeindeprüfungsanstalt Baden-Württemberg, GPA-Mitteilung Bau 1/2007 vom 01.07.2007, Az. 600.535, S. 1-26 
Heilfort T. (2001) Partnerschaftliches Management von Bauablaufstörungen: Mehr Erfolg durch Kooperation? In: Bauwirtschaft, Heft 9/2001, S. 28-29

Heilfort T. (2003) Praktische Umsetzung bauablaufbezogener Darstellung von Behinderungen als Grundlage der Schadensermittlung nach § 6 Nr. 6 VOB/B. In: BauR Heft 4/2003, S. 457-461

IWW (2008): o.V.; Bautagebuch mindert Haftungsrisiken. In: Planungsbüro professionell, Institut für Wissen in der Wirtschaft, Heft 03/2008, S. 14

Kumlehn F. (2004) Bewertung gestörter Bauabläufe der Höhe nach: Geht mit $\S 642$ BGB für Auftragnehmer alles einfacher? In: Baumarkt+ Bauwirtschaft, Heft 9/2004, S. 28-32

Leitzke W. (2006) Haftungsbegründende/haftungsausfüllende Kausalität. In: Schriftenreihe des IBB: Bauablaufstörungen und Entschädigungsberechnung, Institut für Bauwirtschaft und Baubetrieb (Hrsg.), Heft 41/2006, Braunschweig, S. 123-140

Mechning M., Völker B., Mack D., Zielke H. (2014) Ist das Bauzeitlabyrinth ein Irrgarten? In: NZBau - Neue Zeitschrift für Baurecht und Vergaberecht, Heft 02/2014, Beck, Frankfurt a. M., S. 85-92

\section{Online-Dokument}

Bieber M. (2009) Bauablaufstörungen - kurz angesprochen, Seminarvortrag, https://docplayer. org/25941369-Bauablaufstoerungen-kurz-angesprochen-dipl-ing-fh-m-bieber-seminarvortrag. html, Abrufdatum: 01.04.2020

Statistisches Bundesamt (2020) Index der Erzeugerpreise gewerblicher Produkte - Lange Reihen, Inlandsabsatz - Betonstahl, https://www.destatis.de/DE/Themen/Wirtschaft/Preise/Erzeugerpreisindex-gewerbliche-Produkte/_inhalt.html, Wiesbaden, Abrufdatum: 17.04.2020

\section{Urteile}

BGH-Urteil VII ZR 172/86 vom 12.03.1987

BGH-Urteil VII ZR 23/84 vom 27.06.1985

BGH-Urteil VII ZR 286/84 vom 20.02.1986

BGH-Urteil VII ZR 5/91 vom 12.03.1992

BGH-Urteil VII ZR 185/98 vom 21.10.1999

BGH-Urteil VII ZR 224/00 vom 21.03.2002

BGH-Urteil VII ZR 225/03 vom 24.02.2005

BGH-Urteil VII ZR 141/03 vom 24.02.2005

BGH-Urteil VII ZR 201/06 vom 18.12.2008

BGH-Urteil VII ZR 11/08 vom 11.05.2009

BGH-Urteil VII ZR 82/08 vom 10.09.2009

BGH-Urteil VII ZR 152/08 vom 10.09.2009

BGH-Urteil VII ZR 202/09 vom 08.03.2012

BGH-Urteil VII ZR 141/12 vom 08.05.2014

Urteil 20 U 164/84 des OLG Köln vom 14.06.1985

Urteil 17 U 56/00 des OLG Hamm vom 12.02.2004

Urteil 8 U 318/04 des OLG Jena vom 22.03.2005

Urteil 7 U 12/12 des KG Berlin vom 28.05.2013

Beschluss 11 U 70/13 des OLG Köln vom 27.10.2014

Urteil 11 U 102/12 des OLG Brandenburg vom 02.12.2015

Urteil 12 U 222/14 des OLG Brandenburg vom 18.02.2016 